\title{
Engineered Xylose Utilization Enhances Bio-products Productivity in the Cyanobacterium Synechocystis sp. PCC 6803
}

Tai-Chi Lee ${ }^{\mathrm{a}, 1}$, Wei Xiong ${ }^{\mathrm{a}, 1}$, Troy Paddock ${ }^{\mathrm{a}}$, Damian Carrieri ${ }^{\mathrm{a}}$, Ing-Feng Chang ${ }^{\mathrm{b}}$, Hui-Fen Chiu $^{\mathrm{c}}$, Justin Ungerer $^{a}$, Suh-Hang Hank Juo ${ }^{d, *}$, Pin-Ching Maness ${ }^{a, *}$, and Jianping $\mathrm{Yu}^{\mathrm{a}, *}$

a. Biosciences Center, National Renewable Energy Laboratory, Golden, CO, USA.

b. Institute of Plant Biology, National Taiwan University, Taipei, Taiwan.

c. Institute of Pharmacology, Kaohsiung Medical University, Kaohsiung, Taiwan.

d. Department of Genome Medicine, Kaohsiung Medical University, Kaohsiung, Taiwan.

1. These authors contributed equally to this work.

※ Correspondence to:

Jianping.Yu@nrel.gov

Pinching.Maness@nrel.gov

hjuo@kmu.edu.tw

\section{Keywords:}

cyanobacteria; isotopic tracing; photomixotrophic growth; xylose utilization 


\section{Abstract}

Hydrolysis of plant biomass generates a mixture of simple sugars that is particularly rich in glucose and xylose. Fermentation of the released sugars emits $\mathrm{CO}_{2}$ as byproduct due to metabolic inefficiencies. Therefore, the ability of a microbe to simultaneously convert biomass sugars and photosynthetically fix $\mathrm{CO}_{2}$ into target products is very desirable. In this work, the cyanobacterium, Synechocystis 6803 , was engineered to grow on xylose in addition to glucose. Both the $x y / A$ (xylose isomerase) and $x y l B$ (xylulokinase) genes from E. coli were required to confer xylose utilization, but a xylose-specific transporter was not required. Introduction of $x y \mid A B$ into an ethylene-producing strain increased the rate of ethylene production in the presence of xylose. Additionally, introduction of $x y \mid A B$ into a glycogen-synthesis mutant enhanced production of keto acids. Isotopic tracer studies found that nearly half of the carbon in the excreted keto acids was derived from the engineered xylose metabolism, while the remainder was derived from $\mathrm{CO}_{2}$ fixation. 


\section{Introduction}

Concerns regarding global climate change and energy security have intensified research in renewable energy in order to alleviate the overdependence on fossil resources. Conversion of lignocellulosic biomass into fuels and chemicals is widely considered as a sustainable alternative (Adsul et al., 2011; Lange et al., 2012; Vishnu Menon, 2012). Most studies have focused on heterotrophic dark fermentation of biomass-derived sugars into target products (Catalanotti et al., 2013). However, carbon-conversion efficiency in heterotrophic metabolism is an issue. A large portion of carbon in the sugars derived from biomass is lost to $\mathrm{CO}_{2}$ (Humbird et al., 2011) at pyruvate dehydrogenase and at 6-phosphogluconate dehydrogenase, for example. Therefore, a more carbonefficient strategy for the production of biofuels and chemicals is desirable, and may be achieved with a photosynthetic organism that is able to convert both biomass sugars and $\mathrm{CO}_{2}$ into target products (Lam and Lee, 2012). Halophilic, photoautotrophic microorganisms, especially genetically-tractable cyanobacterial strains, hold immense promise as efficient biocatalysts since they can be powered by sunlight to directly convert $\mathrm{CO}_{2}$ into fuels and chemicals, while not competing with food crops for arable land and fresh water (Angermayr et al., 2009; Ducat et al., 2011; Machado and Atsumi, 2012; Yu et al., 2013). Cyanobacteria have been genetically engineered to produce a multitude of fuel intermediates and chemicals such as isoprene (Lindberg et al., 2010), bio-hydrogen

(Baebprasert et al., 2011), ethylene (Ungerer et al., 2012), acetone (Zhou et al., 2012), 2,3butanediol (Savakis et al., 2013), and can convert $\mathrm{CO}_{2}$ to keto acids without cell growth (Carrieri et al., 2012).

Lignocellulosic biomass consists of cellulose (13.2-66.59\%), hemicelluloses (14.8-50\%), and lignin $(10-40 \%)$ (Saha, 2003; Shahzadi et al., 2014). As xylose is the main hydrolysis product of hemicellulose and the second most abundant sugar in Nature after glucose, efficient use of xylose is 
necessary for economic utilization of lignocellulosic biomass. Some microorganisms such as $E$. coli have a native pentose utilization pathway. For xylose to be metabolized, it must be readily transported into the cells by two xylose-specific transport systems: the $x y / E$, a major facilitator superfamily (MFS) transporter acting as a relatively low-affinity xylose/proton symporter (Davis and Henderson, 1987; Jojima et al., 2010), and the $X y / F G H$, a high-affinity xylose transporter complex that belongs to the ATP-binding cassette (ABC) family (Jojima et al., 2010). Inside cells D-xylose is converted by xylose isomerase (XI, encoded by $x y / A)$ to $\mathrm{D}$-xylulose, which is subsequently phosphorylated to xylulose-5-phosphate (X5P) by xylulokinase (XK, encoded by $x y / B)$, where it enters the pentose phosphate pathway (PPP). Genetic engineering by introducing heterologous pentose metabolic pathway genes has enabled xylose utilization in heterotrophic strains (Fan et al., 2011; Hahn-Hagerdal et al., 2007; Jeffries, 2006; Meijnen et al., 2008; Rogers et al., 2007; Toivari et al., 2001; van Maris et al., 2007; Zhang et al., 1995). Recently this approach has also been successful in the cyanobacterium, Synechococcus elongates PCC 7942 (McEwen et al., 2013), although improved biofuel productivity was not reported.

Cyanobacteria exhibit higher solar conversion efficiency compared to plants and eukaryotic algae, and may serve as light-driven microbial catalysts for the production of biofuels and bioproducts (Ducat et al., 2011; Lu, 2010). Nevertheless photosynthesis activity is subject to oscillatory day-night regimes in nature. Concomitant utilization of biomass-derived sugars could broaden the feedstock spectrum and supplement photoautotrophic productivity. Some cyanobacteria are considered strict photoautotrophs, while others can use glucose or fructose under mixotrophic and heterotrophic growth conditions (Ungerer et al., 2008). While no natural strains of cyanobacteria are known to grow on xylose, we provide evidence that conferring xylose-utilization capability to Synechocystis sp. PCC 
6803 (hereafter referred as Synechocystis) by expressing E.coli XylA and XyIB (Fig. 1) enhances bioproduct productivity in a mixotrophic mode compared to only an photoautotrophic mode of growth.

Fig. 1

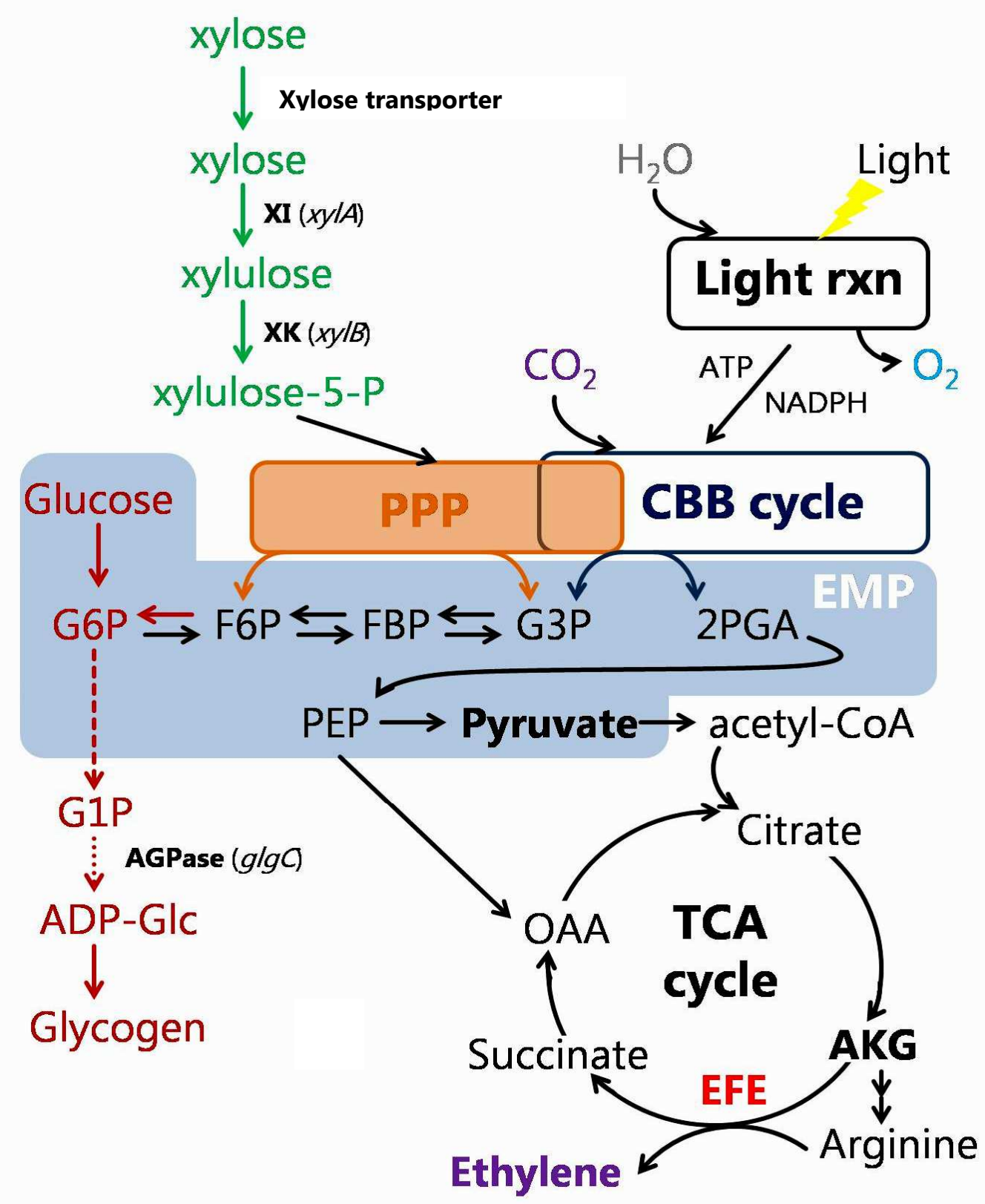

Fig. 1 Overview of the engineered xylose assimilation pathway and central carbon metabolism in Synechocystis 6803. Carbon sources (xylose and/or $\mathrm{CO}_{2}$ ) enter central carbon metabolism in the engineered Synechocystis strains bearing $x y / A B$ genes. Photosynthetic $\mathrm{CO}_{2}$ fixation via the Calvin-Benson-Bassham (CBB) 
cycle produces metabolic intermediate carbon compounds. When xylose enters cells via an endogenous xylose transporter, it is first isomerized into xylulose by xylose isomerase (XI) and then phosphorylated by xylulokinase (XK) to produce xylulose-5-phosphate (X-5-P), an intermediate in the pentose phosphate pathway (PPP). For engineered ethylene production, Ethylene forming enzyme (EFE) catalyzes the conversion of the TCA cycle intermediate AKG into ethylene. AKG and pyruvate production by overflow metabolism occurs in $\triangle g l g C$ strains with blocked glycogen synthesis. Other abbreviations: G1P, glucose-1-phosphate; G3P, glyceraldehyde-3-phosphate; EMP, Embden-Meyerhof-Parnas pathway; rxn, reaction; 2PGA, 2phosphoglycerate; PEP, phosphoenolpyruvate; OAA, oxaloacetic acid; AKG, $\alpha$-ketoglutarate; F6P, Fructose-6phosphate; FBP, Fructose-1.6-bis-phosphate; G6P, Glucose-6-phosphate; and AGPase, ADP glucose pyrophosphorylase.

\section{Materials and Methods}

\subsection{Plasmid construction and strains}

The $x y \mid A, x y / B$ genes, and $x y I A B$ operon from the pentose-competent $E$ coli MG1655 strain were amplified using genomic DNA as template, and a PCR primer-pair containing a Sapl site at the 5' end. They were then ligated to pGEM-T cloning vector (Promega, Co.). To remove an internal Sapl site on the $x y I A$ gene, a silent mutation ( $G$ to $A$ ) was introduced at the 120th nucleotide of the coding region by single primer mutagenesis (Makarova et al., 2000) with Phusion high-fidelity DNA polymerase (NEB, Co), and also using the pGEM-T vector bearing $x y \mid A$ or $x y I A B$ as a template. The full-length fragment containing a $x y \mid A$ or $x y \mid A B$ coding region with the silent mutation was then excised from the vector with Sapl, followed by ligation to the vector fragment of Sapl-digested plasmid, pJU102. This plasmid contains the Sy-efe gene driven by a constitutive psbA promoter (Ungerer et al., 2012), which results in the pJU102/xyIA and pJU102/xyIAB plasmids that can express XI from the $x y \mid A$ gene 
with the silent mutation. Similarly, to generate pJU102/xylB, the $x y / B$ fragment excised from the pGEM-T vector bearing $x y l B$ was used to replace Sy-efe-coding region of pJU102. To generate a control plasmid without the $x y I A B$ sequences, the pJU102/xyIAB was digested with Xmal, followed by self-ligation to delete the $x y \mid A B$ fragment (pJU102/null). The plasmids were transformed into wild-type Synechocystis, and transformants were selected based on spectinomycin resistance. The inserted expression cassette on these plasmids integrated into the s/r0168 neutral site of Synechocystis chromosomes via double homologous recombination, as verified by PCR (Ungerer et al., 2012). The $x y I F G H$ transporter genes were amplified from genomic DNA of E. coli MG1655, using a PCR primer pair containing a Kpnl site at the 5' end and a Spel site at the 3' end, and then ligated to the pGEM-T cloning vector. The Kpnl and Spel double digested $x y I F G H$ fragment from the $x y I F G H$-bearing pGEM-T vector was then used to replace the coding region of the parental plasmid ( $p$ PsbA/xylAB), resulting in the $x y l F G H$-expressing construct (pPsbA/xylFGH). Transformants containing $x y l F G H$ were selected with erythromycin, and targeted integration of the $x y I F G H$ expression cassette at the hoxF locus was confirmed by PCR. Gene insertion at hox $F$ has been shown to impact hydrogen production only in our lab. To introduce the ethylene-production pathway into the $x y \mid A B$ strain, plasmid pJU112 (Ungerer et al., 2012) harboring the expression cassette of the Sy-efe gene was transformed into the $x y I A B$ strain and integrated into the native psbA2 locus, which is a commonlyused recombination site for knock-ins. Transformants were selected based on resistance to spectinomycin and kanamycin, and integration of the Sy-efe expression cassette at the psbA2 locus was verified by PCR (Ungerer et al., 2012). To introduce xylose-utilization capability into a glycogen synthesis mutant $\triangle g l g C$ (Carrieri et al., 2012), pJU102/xylAB was transformed into the $\triangle g l g C$ strain and the resultant $\triangle g l g C / x y \mid A B$ recombinant was selected with spectinomycin and gentamicin. All constructs used in this study were verified by sequencing and are illustrated in Supplementary Fig. 1. All primer sequences used for plasmid construction or PCR analysis are listed in Supplementary 
Table 1. All engineered cyanobacterial strains were selected via serial passages on agar plates, and were completely segregated as verified by PCR and phenotypes.

\subsection{Culture conditions}

Synechocystis was grown on BG11 medium supplemented with $20 \mathrm{mM} \mathrm{NaHCO}, 4.6 \mathrm{~g} / \mathrm{L}$ TES at $30^{\circ} \mathrm{C}$, and in an atmosphere of $5 \% \mathrm{CO}_{2}$ in air under $\sim 50 \mu \mathrm{Em}^{-2} \mathrm{~s}^{-1}$ illumination from cool white fluorescent lamps, with shaking for liquid cultures. The antibiotics, spectinomycin $(25 \mu \mathrm{g} / \mathrm{mL})$, erythromycin $(25 \mu \mathrm{g} / \mathrm{mL})$, gentamicin $(25 \mu \mathrm{g} / \mathrm{mL})$ and kanamycin $(25 \mu \mathrm{g} / \mathrm{mL})$, were used on plates and in liquid cultures, except in media used to test growth and productivity. All assays were conducted with biological triplicates. Growth was measured spectrophotometricallly (WPA Biowave II, Biochrom, USA) as an optical density at $730 \mathrm{~nm}\left(\mathrm{OD}_{730}\right)$.

For heterotrophic and photomixotrophic growth, cells in exponential phase were diluted to $0.1 \mathrm{OD}_{730}$ in BG-11 medium. For light-activated heterotrophic growth (LAHG), liquid cultures incubated in 6-well plates were exposed to illumination at $50 \mu \mathrm{Em}^{-2} \mathrm{~s}^{-1}$ for 1 hour per day and otherwise kept in complete darkness. This condition was modified from prior LAHG (Anderson and Mclntosh, 1991; He and Vermaas, 1998; Kong et al., 2003; Tabei et al., 2009) and improved the consistency of the growth results. The chlorophyll content was determined by measuring the absorbance at $666 \mathrm{~nm}$ as described before (Gao et al., 2009). To test mixed sugars utilization, Synechocystis was cultivated under photomixotrophic conditions in 6-well plates under continuous illumination at $50 \mu \mathrm{Em}^{-2} \mathrm{~s}^{-1}$ with $5 \% \mathrm{CO}_{2}$. Cells in the exponential phase were diluted to $0.6 \mathrm{OD}_{730}$ in $\mathrm{BG}-11$ medium, supplemented with appropriate sugars. Xylose in the medium was measured with High-Performance Liquid Chromatography (Agilent Technologies 1200 series HPLC). Glucose in the medium was determined using a glucose assay kit (Sigma-Aldrich). 
For the nitrogen starvation study related to pyruvate and a-ketoglutarate (AKG) production, TES was removed from BG11 recipe (1X BG11-TES), since co-elution of TES and pyruvate in the HPLC interferes with pyruvate quantitation. In the $1 \mathrm{X}$ BG11-TES-N medium sodium nitrate $\left(\mathrm{NaNO}_{3}\right)$ was replaced with equal moles of sodium chloride $(\mathrm{NaCl})$. For the isotope tracer experiment, "1X BG11TES-N-bicarbonate" medium was used. Here unlabeled bicarbonate was omitted, and ${ }^{13} \mathrm{C}$ bicarbonate was added to the culture to start the labeling process.

\subsection{Pyruvate and AKG production assay}

The $\triangle g l g C$ and $\triangle g l g C / x y \mid A B$ strains were cultivated in $1 X$ BG11 medium to an $\mathrm{OD}_{730}$ of 1.0. Cells were spun down by centrifugation at $3000 \mathrm{~g}$ for 10 minutes. Cell pellets were re-suspended in either 1X BG11-TES medium or 1X BG11-TES-N medium, in the presence or absence of $20 \mathrm{mM}$ xylose. The initial $O D_{730}$ of each culture was then adjusted to 0.8 . Cultures were shaken at $220 \mathrm{rpm}$ under 50 $\mu \mathrm{Em}^{-2} \mathrm{~s}^{-1}$ illumination and sampled over time for growth measurements. For AKG and pyruvate measurements, culture samples were collected, and the medium was separated from cells by table top centrifugation. The supernatant was then filtered through $0.2 \mu \mathrm{M}$-diameter nylon membrane (Acrodisc $^{\circledR}$ ) and analyzed by HPLC (Agilent Technologies1200 series). One hundred $\mu \mathrm{L}$ samples were injected into HPLC column (Bio-Rad Aminex HPX-87H), eluted with $5 \mathrm{mM}$ sulfuric acid at a flow rate of $0.6 \mathrm{ml} / \mathrm{min}$, and the output detected with a UV detector at $210 \mathrm{~nm}$.

\subsection{Ethylene production assay}

Cultures of the $x y \mid A B / e f e$ strain were grown photoautotrophically to exponential phase, then concentrated $5 X$ for the ethylene production assay, as described in (Ungerer et al., 2012), except that $50 \mathrm{mM}$ of xylose was added to half of the sealed tubes. The tubes were then shaken under the 
tested light intensities or in darkness for 12 hours.

\subsection{Isotope tracer analysis}

The $\triangle g l g C / x y \mid A B$ strain was grown in $1 \mathrm{X} B G 11$ medium to an $\mathrm{OD}_{730}$ of 1.0 . Cells were spun down by centrifugation at $3000 \mathrm{~g}$ for 10 minutes, and the pellets were re-suspended in BG11-TES-Nbicarbonate medium at an $\mathrm{OD}_{730}$ of 0.8 in the presence or absence of $20 \mathrm{mM}$ unlabeled $\mathrm{D}$-xylose. Isotope labeling was started with the addition of $20 \mathrm{mM}{ }^{13} \mathrm{C}$-bicarbonate. Sealed flasks (head space volume: culture volume $=5: 1$ ) were used to avoid introducing spurious $\mathrm{CO}_{2}$ from the air, and they were shaken at $220 \mathrm{rpm}$ under $50 \mu \mathrm{Em}^{-2} \mathrm{~s}^{-1}$ illumination for 24 hours. Aliquots of the cultures were then filtered for the analysis of AKG and pyruvate in the medium. Labeling patterns of AKG and pyruvate were analyzed by reverse-phase, ion-pairing liquid chromatography (LC) coupled to electrospray ionization (ESI) mass spectrometry using a procedure similar to (Luo et al., 2007). A Synergi Hydro-RP (C18) 250 mm x 1 mm column (Phenomenex, Aschaffenburg, Germany) was used for chromatographic separation. The mobile phase was comprised of eluent A (10 mM tributylamine in water with $3 \%$ methanol [pH adjusted to 4.95 with $15 \mathrm{mM}$ acetic acid]) and eluent $\mathrm{B}$ (methanol) under the following linear gradient conditions: $t=0,0 \% \mathrm{~B} ; t=5 \mathrm{~min}, 0 \% \mathrm{~B} ; t=20 \mathrm{~min}, 20 \% \mathrm{~B} ; t=35$ $\min , 20 \% \mathrm{~B} ; t=60 \mathrm{~min}, 65 \% \mathrm{~B} ; t=65 \mathrm{~min}, 95 \% \mathrm{~B} ; t=75 \mathrm{~min}, 95 \% \mathrm{~B} ; t=80 \mathrm{~min}, 0 \% \mathrm{~B}$. The injection volume was $50 \mu \mathrm{l}$, and the column was equilibrated before each injection by pre-running $100 \%$ eluent A for 3 minutes. The flow rate was $0.05 \mathrm{ml} / \mathrm{min}$. Metabolites were detected using an Agilent lon Trap mass spectrometer (LC/MSD Trap SL) operated in the negative-ion mode.

To analyze isotope labeling patterns of AKG and pyruvate, a mass isotopomer distribution vector, $M D V_{\alpha}$, was assigned according to (Nanchen et al., 2007). 


$$
\operatorname{MDV}_{\alpha}=\left[\begin{array}{c}
\left(\mathrm{m}_{0}\right) \\
\left(\mathrm{m}_{1)}\right. \\
\vdots \\
\left(\mathrm{m}_{\mathrm{n}}\right)
\end{array}\right] \quad \sum_{\mathrm{i}=0}^{\mathrm{n}} \mathrm{m}_{\mathrm{i}}=1
$$

where $m_{0}$ was the fractional abundance of molecules with mono-isotopic mass and $m_{i>0}$ was the abundance of fragments with heavier masses. The LC-MS data were corrected for the naturally occurring isotopes of oxygen $(\mathrm{O})$, hydrogen $(\mathrm{H})$ and carbon $(\mathrm{C})$ atoms using a correction matrix (Eq. 2) (Nanchen et al., 2007).

$$
\mathrm{MDV}_{\alpha}{ }^{*}=\mathrm{C}_{\mathrm{corr}, \mathrm{COH}^{-1}} \cdot \mathrm{MDV}_{\mathrm{a}}
$$

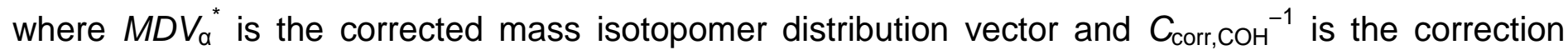
matrix. The resulting $M D V_{\alpha}^{*}$ values were then used to assess the fractional labeling enrichment of AKG and pyruvate using Equation 3.

$F L=\frac{\sum_{i=0}^{n} i \cdot m i}{n \cdot \sum_{i=0}^{n} m i}$

where $n$ represents the number of carbon atoms in AKG or pyruvate and $i$ is the mass isotopomer.

\section{Results}

\subsection{Xylose utilization}

The common laboratory Synechocystis wild-type (WT) can grow on glucose but not on xylose, and genes encoding xylose-specific transporters and the key catabolizing enzymes XI and XK were not found in its genome. In order to identify genes necessary to confer xylose utilization in Synechocystis, we introduced E. coli genes encoding a xylose transporter (encoded by $x y / F G H$ ) and two xylosemetabolizing enzymes (encoded by $x y \mid A B$ ). The expression constructs containing the coding regions 
of $x y \mid A B$ and $x y / F G H$ were generated (Supplement, Fig. 1). They were designed to constitutively express the catalytic enzymes (XI and $\mathrm{XK})$ and/or the transporter after targeted integration into the cyanobacterial genome. The plasmids were transformed into the WT, and the resultant strains $x y \mid A B / F G H$ and $x y I A B$ were tested for heterotrophic growth on xylose. Synechocystis cannot grow on sugar supplementation in complete darkness unless given a daily pulse of white light (light-activated heterotrophic growth or LAHG; (Anderson and McIntosh, 1991). Accordingly, we cultured the cells on xylose in the dark with daily one-hour illuminations at $50 \mu \mathrm{Em}^{-2} \mathrm{~s}^{-1}$. The $x y / A B$ LAHG cultures showed a slower growth rate $\left(\sim 0.12 \mathrm{OD}_{730}\right.$ per day) over the first 6 days with $5-50 \mathrm{mM}$ xylose, followed by a faster growth rate $\left(\sim 0.7 \mathrm{OD}_{730}\right.$ per day) on $10 \mathrm{mM}$ xylose (Fig. 2 A). In contrast, neither the WT nor cells expressing individual $x y / A$ or $x y / B$ gene were able to grow on xylose under LAHG (data not shown), indicating that both $x y I A$ and $x y / B$ are required for xylose metabolism. Growth on glucose was also tested as the positive control of LAHG. As expected, the $x y / A B$ strain exhibited identical growth to the control strain (wild-type) on $5.6 \mathrm{mM}(0.1 \%)$ glucose (data not shown), displaying a faster rate of $\sim 0.25 \mathrm{OD}_{730}$ per day in the first 6 days comparing to growth on xylose. Similar growth was observed in the $x y \mid A B$ strain and the $x y \mid A B / F G H$ strain with xylose concentration up to $10 \mathrm{mM}$ (Fig. 2 B).

The $x y \mid A B$ strain grew under LAHG conditions for 9 days with up to $50 \mathrm{mM}$ xylose, while the null control strain did not grow (Fig. 2 A and B). The data indicate that the engineered $x y / A B$ and $x y / A B / F G H$ strains were able to consume xylose as the sole carbon source to sustain growth in the dark. It also suggests that the transport is not rate-limiting for xylose catabolism at concentrations below $10 \mathrm{mM}$ under LAHG condition (Fig. 2 B). Finally, the chlorophyll content increased in parallel with culture density and also achieved a maximal value at day 9 in the cultures supplemented with 10 mM xylose (Fig. 2 C). 
Fig. 2 A

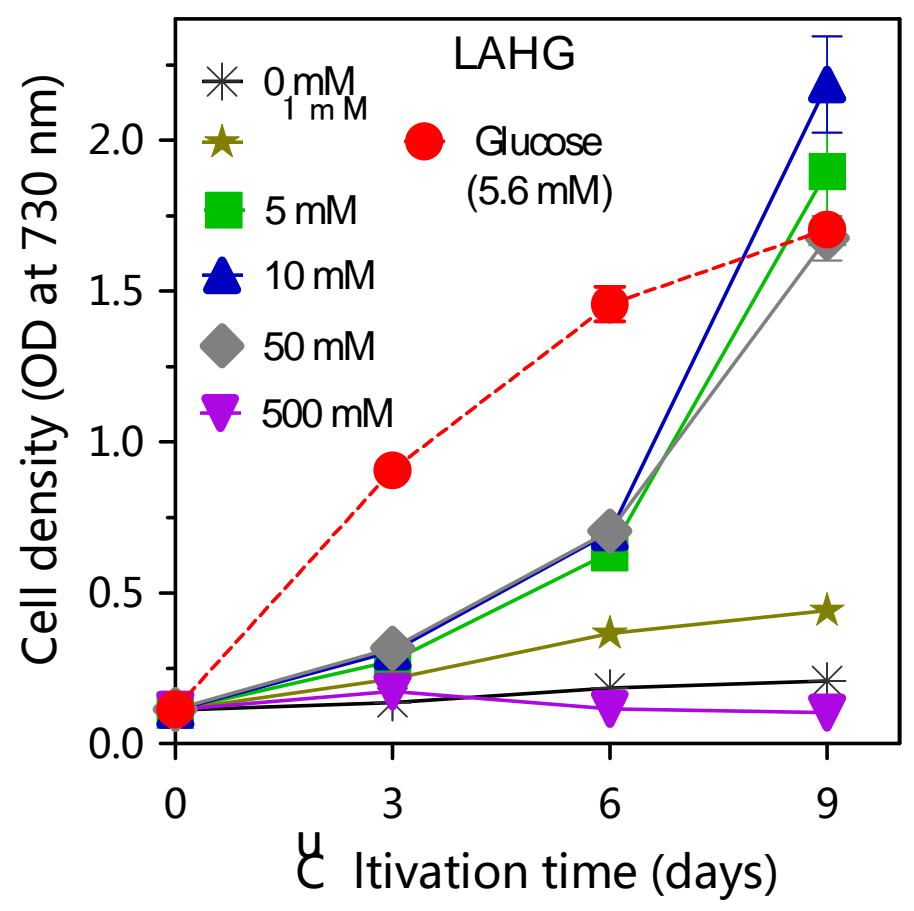

B

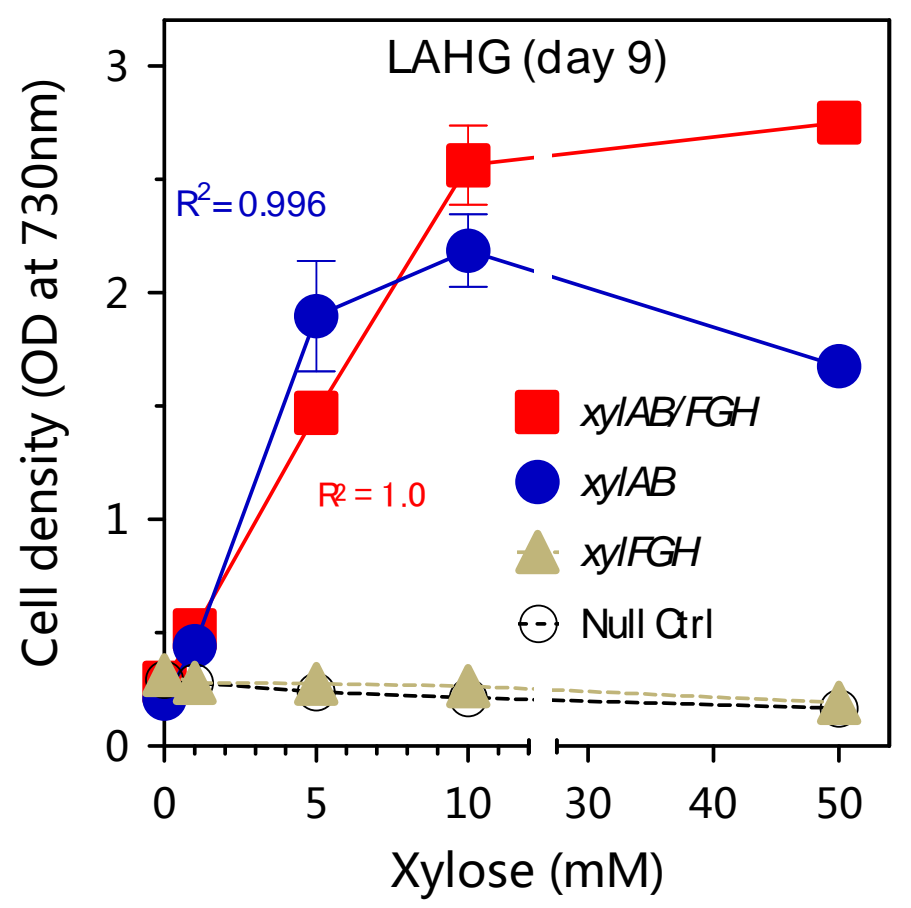




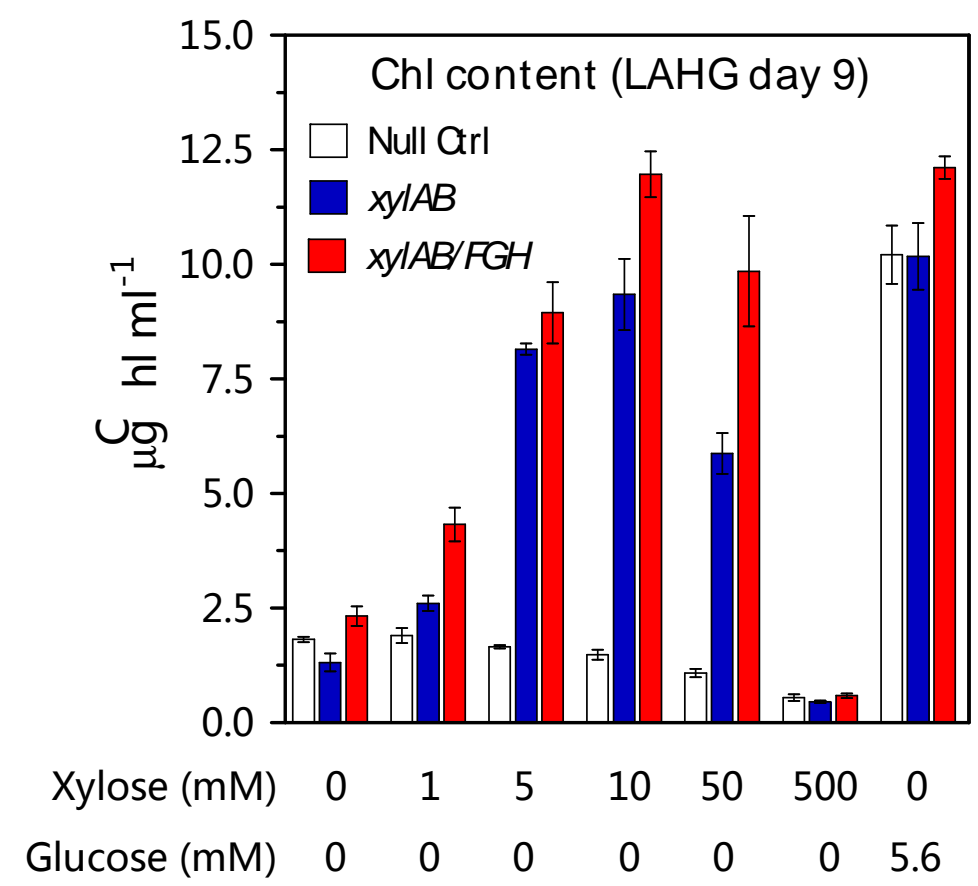

Fig 2. Light-activated heterotrophic growth (LAHG) of the engineered Synechocystis 6803 strains on xylose. Cultures of Synechocystis cells bearing $x y I A B$ and/or $x y / F G H$ genes, or pJU102/null control (null Ctrl) grown photoautotrophically at late log phase were collected. They were then grown from a starting cell density of $0.1 \mathrm{OD}_{730}$ in $\mathrm{BG}-11$ medium in the dark, but with light exposure $\left(50 \mu \mathrm{Em}^{-2} \mathrm{~s}^{-1}\right)$ for 1 hour per day. (A) Growth profile of $x y I A B$-bearing Synechocystis cells under LAHG conditions with addition of the indicated concentrations of xylose. Growth on glucose was tested as the positive control of LAHG. (B) OD $\mathrm{D}_{730}$ at day 9 as a function of the amount of added xylose. Linear correlation between $O D_{730}$ and added xylose ranging from 0 to 5 or 0 to $10 \mathrm{mM}$ was determined for $x y / A B$ or $x y / A B / F G H$ strain respectively. (C) Chlorophyll content at day 9 as a function of the amount of added sugar. All experiments were performed in 6-well plates with shaking at $30^{\circ} \mathrm{C}$. Data shown are means \pm standard deviations from three independent experiments. 
Expression of the $x y I A B$ genes in Synechocystis enabled xylose utilization without a heterologous xylose transporter, implying the existence of an unknown endogenous xylose transport system. HPLC analysis was used to measure xylose uptake by the recombinant strain. We found that $2.5 \mathrm{mM}$ xylose in the medium was completely consumed by the $x y I A B$ strain under LAHG within 9 days (Fig. $3 \mathbf{A}$ ), whereas substantial xylose remained in the medium of the null control strain. Additionally, growth and xylose consumption under photomixotrophic condition were also investigated. Xylose completely disappeared from the $x y I A B$ culture medium within 48 hours, during which the culture grew from 0.1 to $\sim 1.23 \mathrm{OD}_{730}, \sim 0.6 \mathrm{OD}_{730}$ over control strain which did not consume any xylose (Fig. 3 B). Thus xylose uptake and utilization in this strain is much more efficient under photomixotrophic conditions than LAHG. The growth of $x y / A B$ strain was inhibited by high xylose loading (500 mM) under LAHG (Fig. 3 A), while completely tolerating up to 200 to $300 \mathrm{mM}$ xylose in diurnal cycles (Supplement, Fig. 2). Taken together, our results show that the introduction of both $x y / A$ and $x y / B$ genes is necessary and sufficient to enable xylose utilization by Synechocystis under either LAHG or photomixotrophic growth conditions. 
Fig. $3 \quad A$

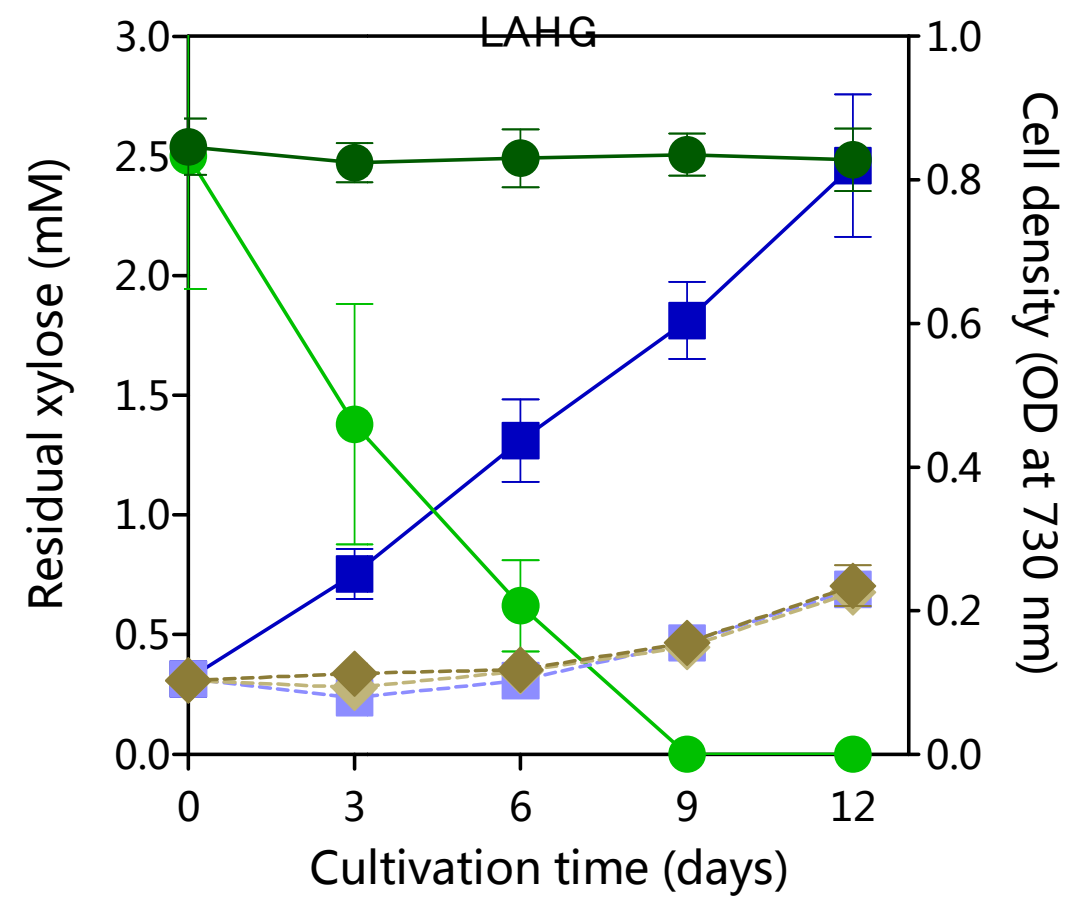

[xylose]: Ctrl (+xylose)

[xylose]: $x y / A B$ (+xylose)

$\square$ OD: $x y / A B$ (+xylose)

OD: $x y / A B$ (-xylose)

OD: Ctrl (+xylose)

OD: Ctrl (-xylose) 


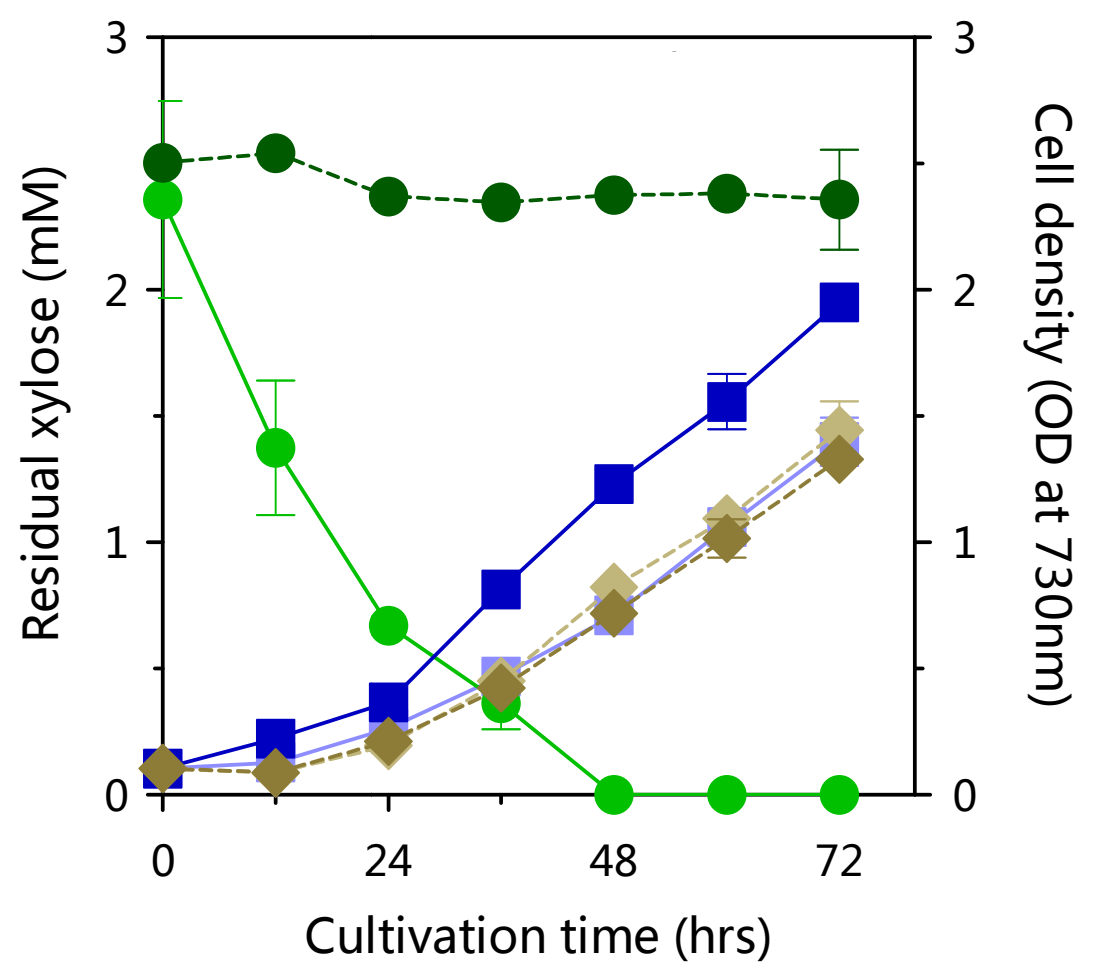

Fig 3. Xylose assimilation by the xylAB-bearing Synechocystis strain. (A) Growth and xylose consumption kinetics of the $x y I A B$ strain under LAHG conditions in the presence of $2.5 \mathrm{mM}$ xylose. The level of xylose in the medium was measured at the indicated times by HPLC (left-Y axis), and cell density was represented as $\mathrm{OD}_{730}$ (right-Y axis). (B) Growth and xylose consumption of $x y \mid A B$ strain under photomixotrophic conditions. 
The xylose-adapted cultures grown photoautotrophically to the late-log growth phase were collected and then re-incubated at an initial cell density of $0.1 \mathrm{OD}_{730}$ in $\mathrm{BG}-11$ medium under continuous illumination (50 $\mathrm{Em}^{-2} \mathrm{~s}^{-1}$ ) in the presence of $2.5 \mathrm{mM}$ xylose at $30^{\circ} \mathrm{C}$. Residual xylose in the medium and cellular growth were determined by HPLC (left-Y axis) and measurement of $\mathrm{OD}_{730}$ (right-Y axis), respectively. All experiments were performed in 6 -well plates with shaking at $30^{\circ} \mathrm{C}$. Data shown are means \pm standard deviations from three independent experiments.

\subsection{Pattern of mixed-sugar utilization}

Certain fermentative microorganisms are known to utilize sugars sequentially with a preference of glucose over xylose (Jojima et al., 2010; Kim et al., 2012). In order to investigate if the $x y \mid A B$ strain also has such preference, photomixotrophic cultures with a starting cell density of $0.6 \mathrm{OD}_{730}$ were tested with $10 \mathrm{mM}$ glucose or xylose, or an equal molar mixture totaling $20 \mathrm{mM}$. As shown in Fig. 4 A, xylose was not taken up at significant amounts during the initial 18-24 hours of cultivation when glucose was abundant. Moreover, glucose uptake was not affected by the presence of xylose in the medium. After 18-24 hours, as the glucose concentration continued to drop, xylose uptake was observed, and the culture density increased gradually to levels comparable to or higher than observed with glucose alone (Fig. 4 B). These observations indicate the $x y \mid A B$ strain preferentially consumes glucose over xylose. Xylose consumption started at around 18-24 hours when glucose to xylose molar ratio decreased to approximately 1:2 (average $4.43 \mathrm{mM}$ for glucose and $8.95 \mathrm{mM}$ for xylose), similar to findings in E. coli (Lawford and Rousseau, 1994). Additionally, similar to the growth profile in LAHG, this strain also grew faster on glucose alone in the mixotrophic mode (Fig. 4 B), reaching $\sim 5.07$ final $O D_{730}$ comparing to in xylose alone with $\sim 3.63 \mathrm{OD}_{730}$ in 48 hours. 
Fig. $4 \quad \mathrm{~A}$

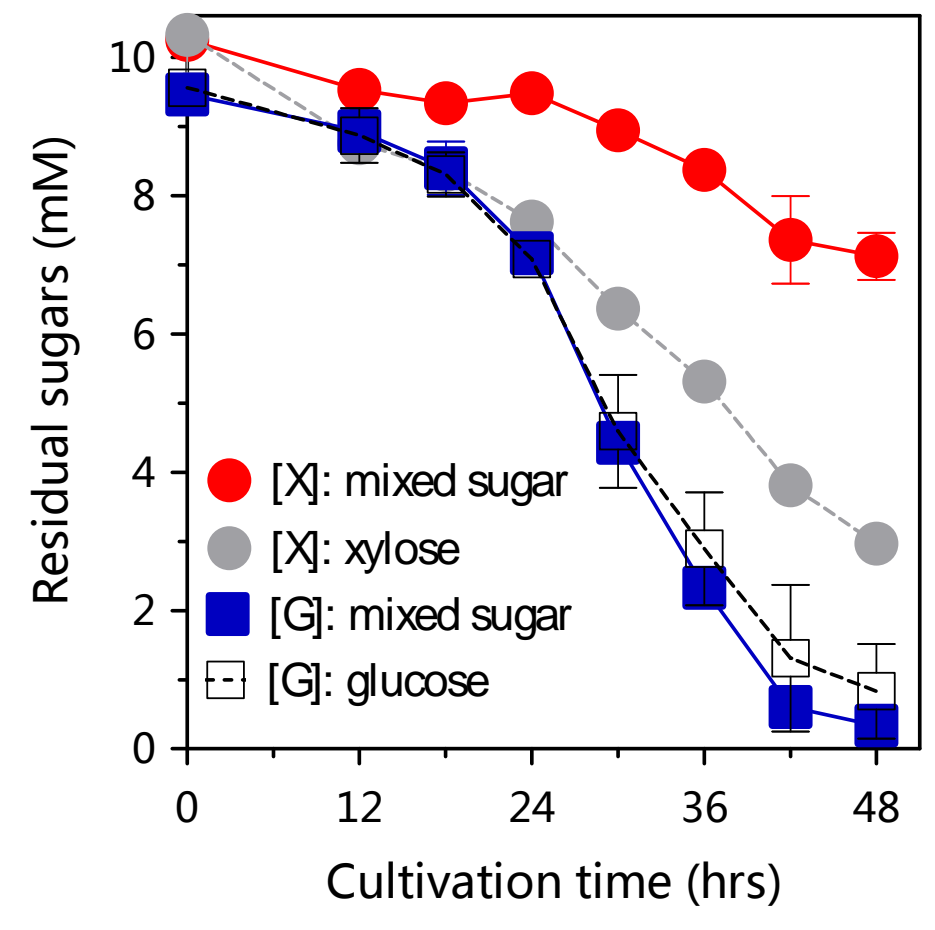

B

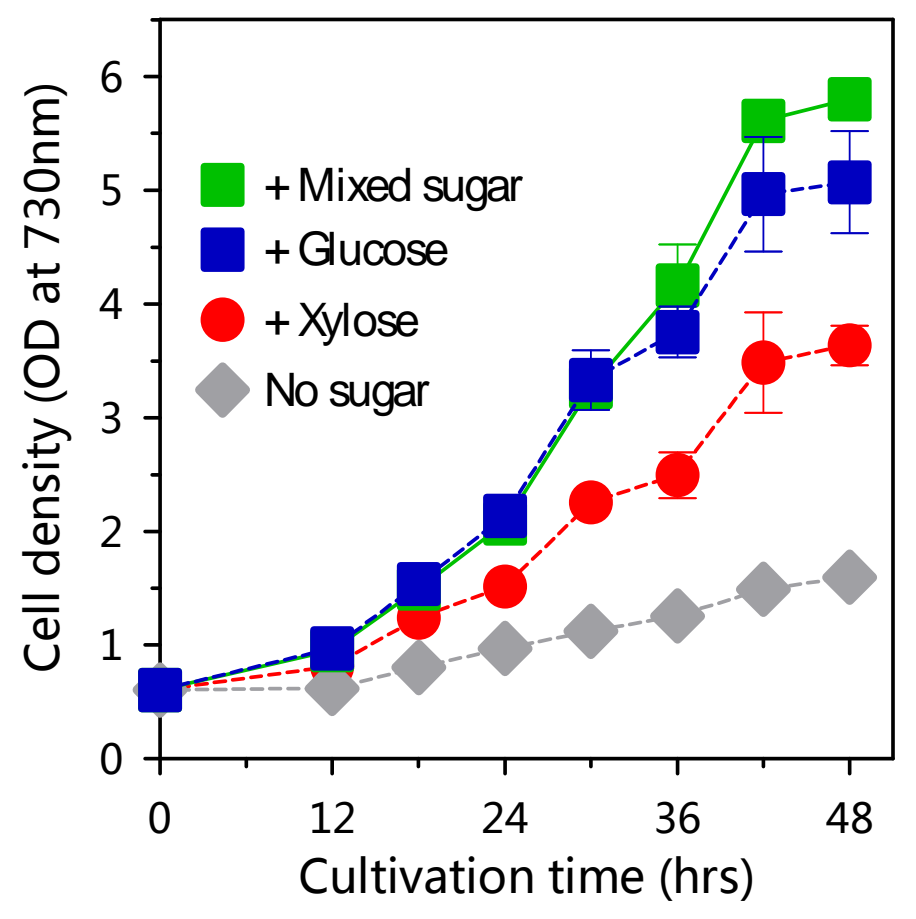


Fig.4. Utilization pattern of sugar mixtures under photomixotrophic conditions by engineered Synechocystis $x y \mid A B$ cells. The $x y \mid A B$ cells harvested at exponential growth phase $\left(0.6 O_{730}\right)$ were inoculated into $1 X$ BG11 medium, either in the presence of mixed sugars (10 mM xylose and $10 \mathrm{mM}$ glucose), $10 \mathrm{mM}$ xylose only or $10 \mathrm{mM}$ glucose only. (A) Sugar consumption by $x y / A B$ strain was examined at different time points. The concentrations of residual xylose and glucose were analyzed by HPLC and glucose assay (HK) kits, respectively. The symbol $[X]$ represents the concentration of residual xylose in cultures containing mixed sugars (filled red circles) or xylose alone (filled gray circles). [G] represents the concentration of residual glucose in culture containing mixed sugars (plotted as filled blue rectangles) or glucose alone (plotted as open diamonds). (B) The cell density of each culture grown in different sugar medium (mixed sugars, glucose or xylose alone) was determined by measuring the $\mathrm{OD}_{730}$ at the indicated time points. All experiments were performed in 6 -well plates with shaking at $30^{\circ} \mathrm{C}$ under continuous illumination $\left(50 \mu \mathrm{Em}^{-2}\right.$ $\left.s^{-1}\right)$. Data shown are means \pm standard deviations from three independent experiments.

\subsection{Conversion of xylose to ethylene}

To investigate the potential of our cyanobacterial strains with an engineered xylose utilization capability for converting biomass sugars to useful products, we chose ethylene and keto-acid production as test cases. Strains of Synechocystis expressing the heterologous ethylene forming enzyme (EFE) were recently shown to continuously convert $\mathrm{CO}_{2}$ into ethylene by photosynthesis. Ethylene is produced by EFE using a-ketoglutarate (hereafter referred as AKG, also known as 2oxoglutarate) and arginine as substrates (Eckert et al., 2014; Ungerer et al., 2012).

The $x y I A B$ strain was further engineered to incorporate a copy of the Sy-efe gene (Ungerer et al., 2012), resulting in a double knock-in $x y l A B /$ efe strain. This strain grew as well as the single 
mutants and the WT under standard photoautotrophic growth conditions (data not shown). Using this strain, we tested whether the introduction of xylose-utilization capability could increase ethylene productivity. As previously observed with the efe strain but without xylose utilization, ethylene production was a function of light intensity (Fig. 5). In contrast, xylose utilization increased culture growth by $40 \%, 35 \%, 30 \%$ and $45 \%$ respectively and ethylene production by $27 \%, 19 \%$, $64 \%$ and $32 \%$ under light intensities of $0,5,40$ and $110 \mu \mathrm{Em}^{-2} \mathrm{~s}^{-1}$ for 12 hours (Fig. 5). The increase in ethylene production was apparently linked to increased cell density, rather than increased productivity on a per cell basis.

Fig. 5

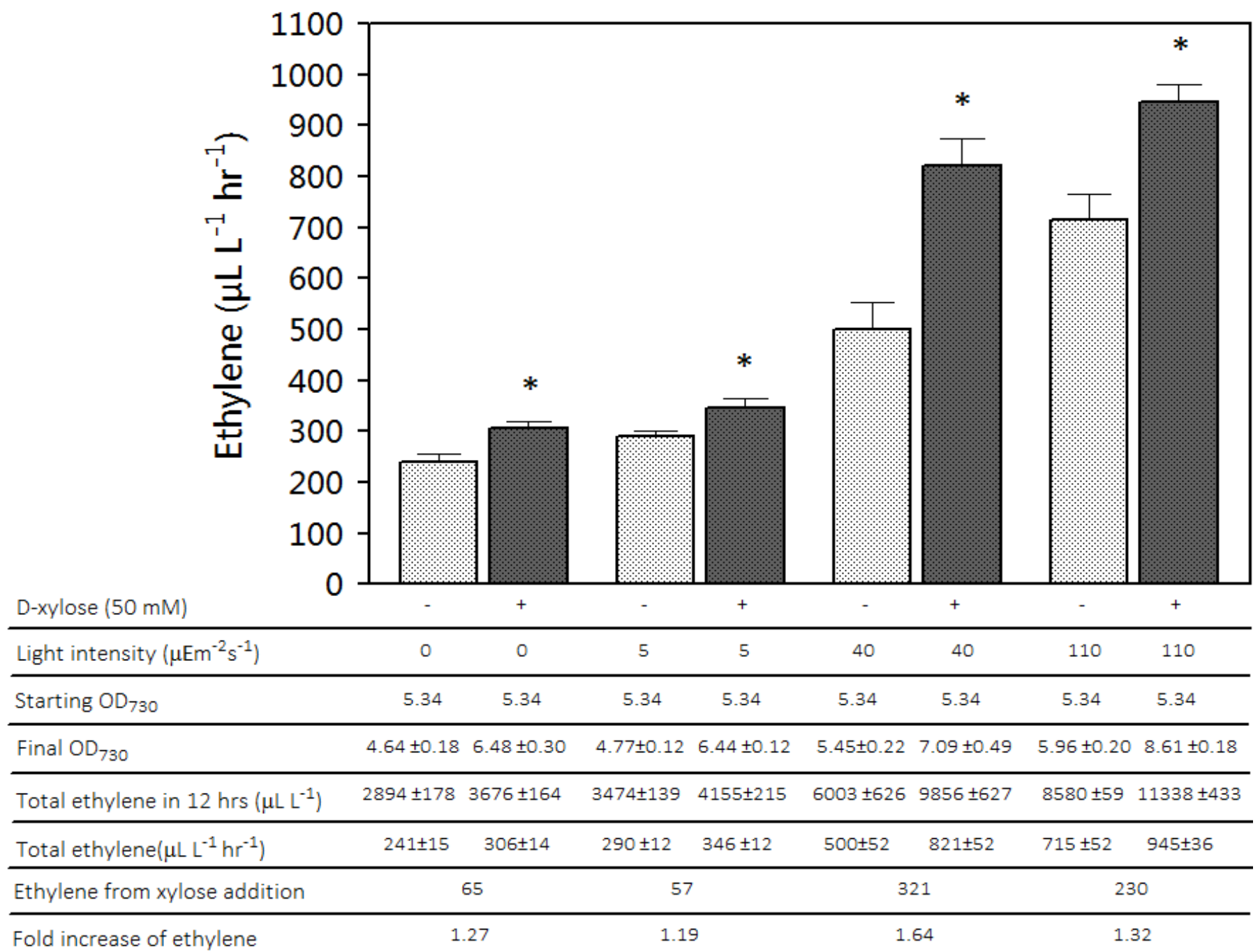


Fig. 5 Ethylene production by $x y I A B / e f e$ strain under photomixotrophic conditions. Cells from late exponential phase $\left(\sim 1.0 \mathrm{OD}_{730}\right)$ were concentrated to approximately $\sim 5.3 \mathrm{OD}_{730}$. The cells $(2 \mathrm{ml})$ were then incubated in sealed 13-ml Hungate tube with or without the addition of $50 \mathrm{mM}$ xylose for 12 hours while shaking at a $60^{\circ}$ angle under the indicated light intensities. Ethylene production was quantified at the end of the incubation period by extracting a gas sample from the headspace through the septum and analyzing it using a HP 5980 gas chromatograph. Detailed calculation of ethylene production was described in Materials \& Methods. Extra ethylene produced from xylose utilization was calculated as the difference between the amount of ethylene produced under xylose addition and that from the no-sugar-added control under the same light intensity (in $\mu \mathrm{L} \mathrm{L}^{-1} \mathrm{hr}^{-1}$ ). Fold increase of ethylene was calculated as the total ethylene produced under xylose addition divided by that produced without xylose. Data shown are means \pm standard deviations from three independent experiments. Significant differences between the values of the xylose-added group and the control group at the identical illumination were determined by Student's $t$ test. Asterisk $(*), p<0.01$ compared to the control group (without xylose).

\subsection{Xylose utilization enhances production of pyruvate and AKG}

To further evaluate if the engineered xylose catabolism could enhance cyanobacterial productivity and carbon utilization, we studied xylose metabolism in a system where the majority of the carbon flux could be rerouted toward desired target products. We recently reported that a glycogen synthesis mutant $\triangle g l g C$ halted growth and excreted pyruvate and AKG into the medium upon nitrogen starvation under photoautotrophic conditions (Carrieri et al., 2012). Excretion of these keto acids from similar mutants was also reported under mixotrophic growth conditions with glucose supplementation (Grundel et al., 2012). We introduced the $x y / A B$ genes into the $\triangle g l g C$ mutant to generate the $\triangle g l g C / x y l A B$ strain. Growth of this double mutant was indistinguishable 
from the single $\Delta g l g C$ mutant under normal photoautotrophic conditions (Figure $6 \mathrm{~A}$ ), and the level of keto acids secreted into the medium was negligible (see below). With $20 \mathrm{mM}$ xylose supplementation, the $\triangle g l g C / x y \mid A B$ strain grew slightly faster in mixotrophic mode and excreted more keto acids than the single $\Delta g l g C$ mutant. This observation contrasts with the previously reported growth sensitivity of similar Synechocystis mutants to photomixotrophic growth with glucose (Grundel et al., 2012), and this may be related to the different sugar metabolic pathways used. In 96 hours, an average of $4263 \mu \mathrm{M}$ of pyruvate and $839 \mu \mathrm{M}$ of AKG were detected in the medium of the double mutant supplemented with xylose, far more than the controls (Fig. 6 B, C), demonstrating that xylose utilization does improve keto acid productivity even under nitrogen replete conditions. Under nitrogen starvation $(-\mathrm{N})$ conditions, growth halted as expected in both the $\triangle g \lg C$ and $\triangle g \lg C / x y \mid A B$ strains, with or without xylose supplementation (Fig. 6 A), and keto acids were released into the medium (Fig. 6 D, E). Xylose utilization by $\Delta g / g C / x y l A B$ led to the highest rates of keto acid production (190 $\mu \mathrm{M}$ per hour for pyruvate and $95 \mu \mathrm{M}$ per hour for AKG in the first 24 hours). These rates were significantly higher than those in control medium (-N) without xylose addition (105 $\mu \mathrm{M}$ per hour for pyruvate and $40 \mu \mathrm{M}$ per hour for $\mathrm{AKG}$ ) and those produced by $\Delta g l g C$ single mutant ( $78 \mu \mathrm{M}$ per hour for pyruvate and $\sim 40 \mu \mathrm{M}$ per hour for AKG with xylose addition). The peak titers of $6921 \mu \mathrm{M}$ for pyruvate and $\sim 2889 \mu \mathrm{M}$ for AKG were observed in the $\triangle g l g C / x y \mid A B$ in xylose supplemented -N medium (Fig. 6 D, E), where the residual xylose was $10.84 \pm 2.02 \mathrm{mM}$ after 5 days. These data demonstrate that xylose utilization significantly enhanced productivities of both pyruvate and AKG under nitrogen starvation (-N) conditions. The carbon utilized from xylose and that in the excreted keto acids were calculated over time (Fig. 6 F), and during the first few days, the carbon in the products was more than that in the sugar feedstock consumed. This demonstrates that mixotrophic metabolism can enhance (compared to 
Fig. $6 \quad A$

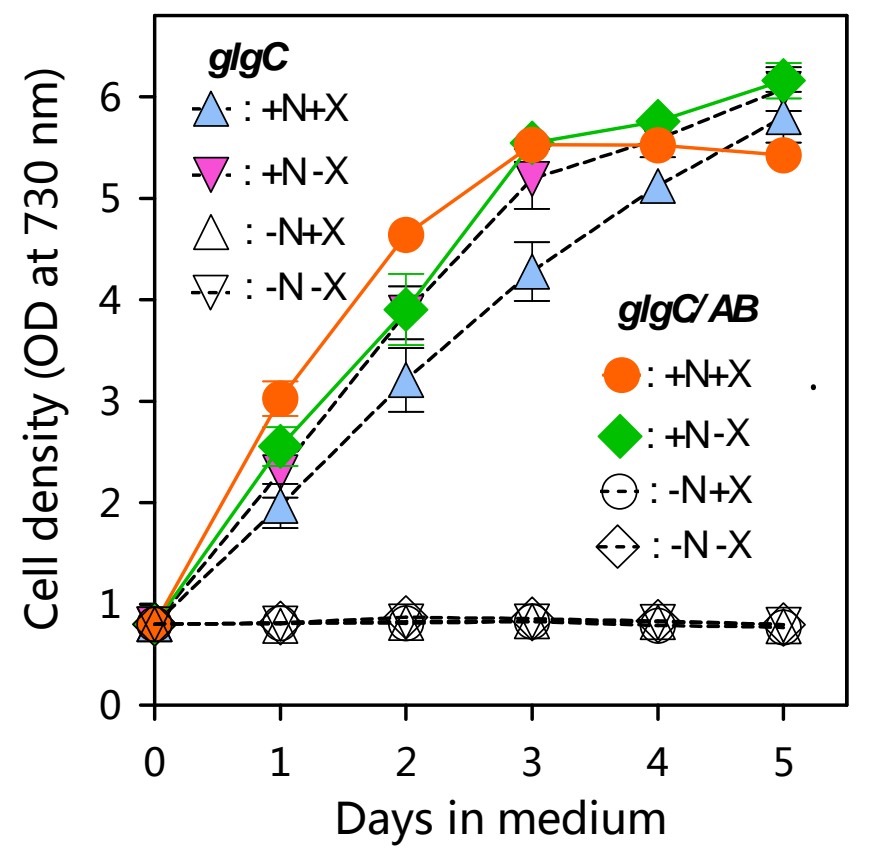

B

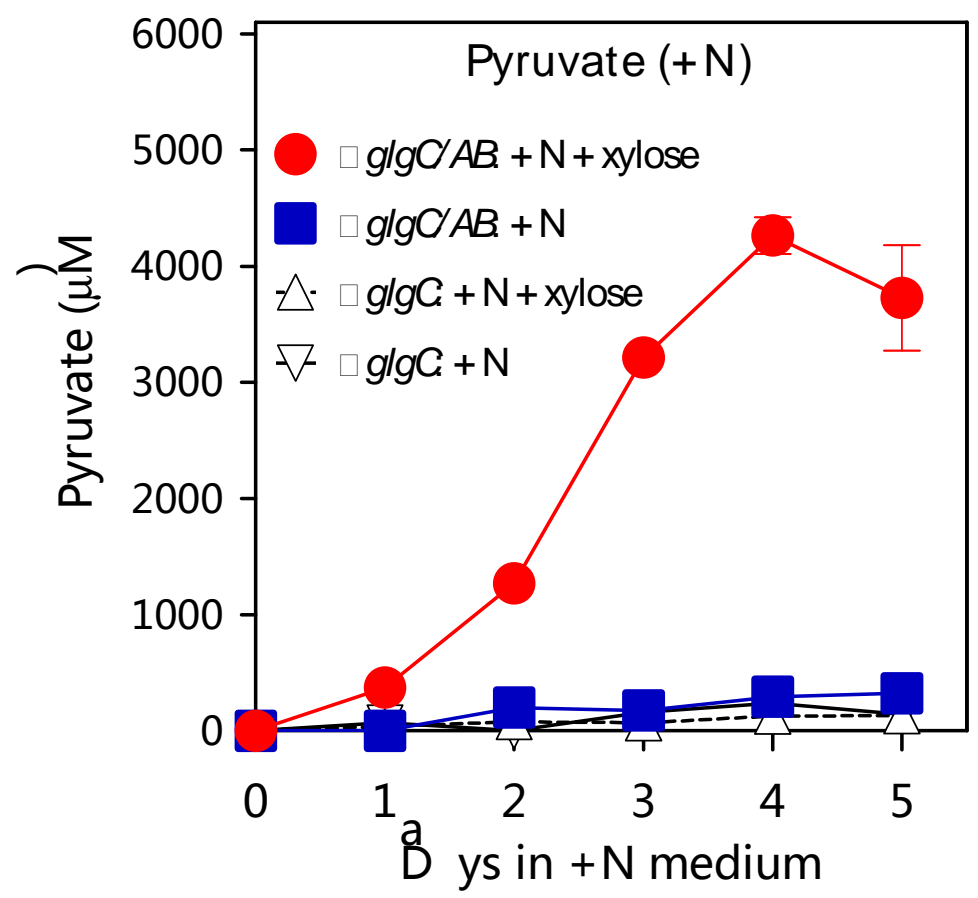


C
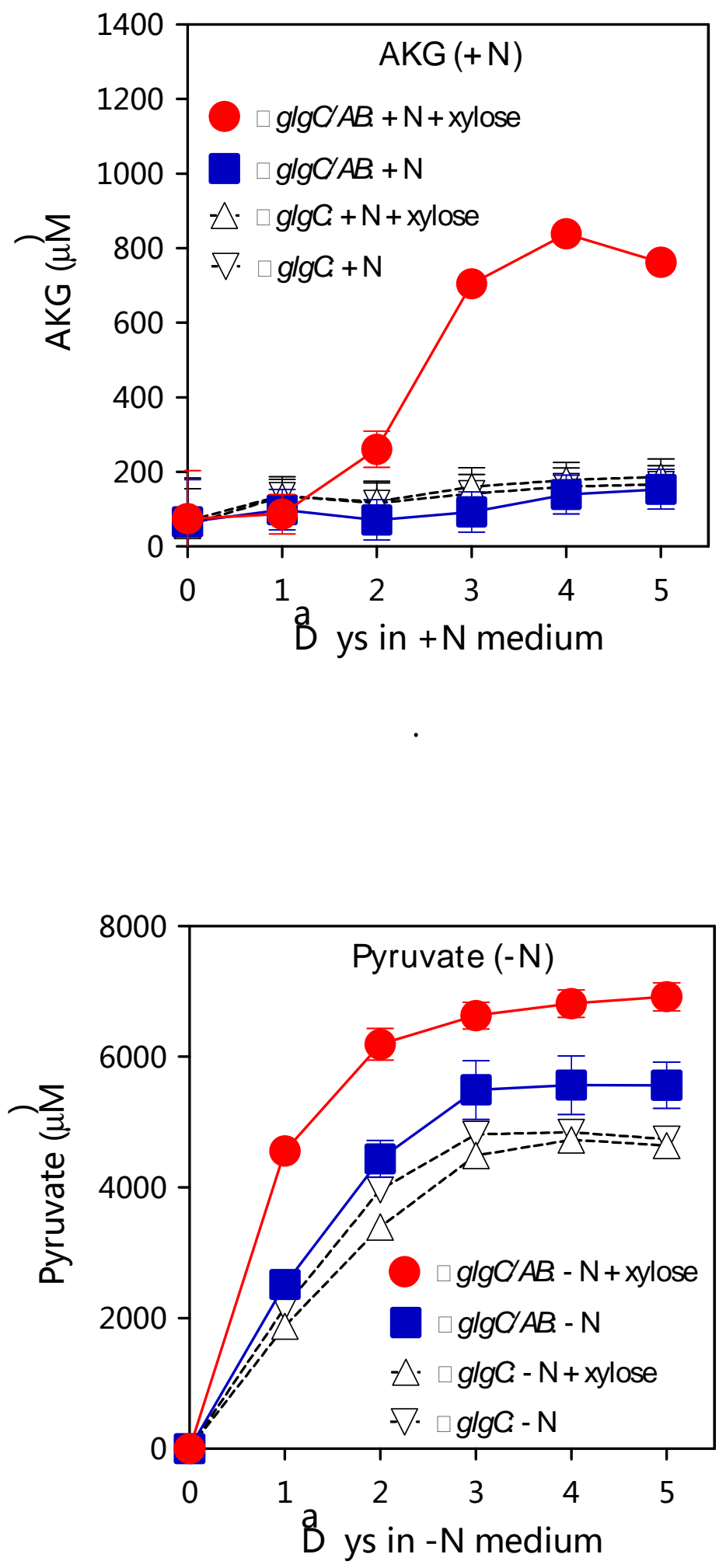


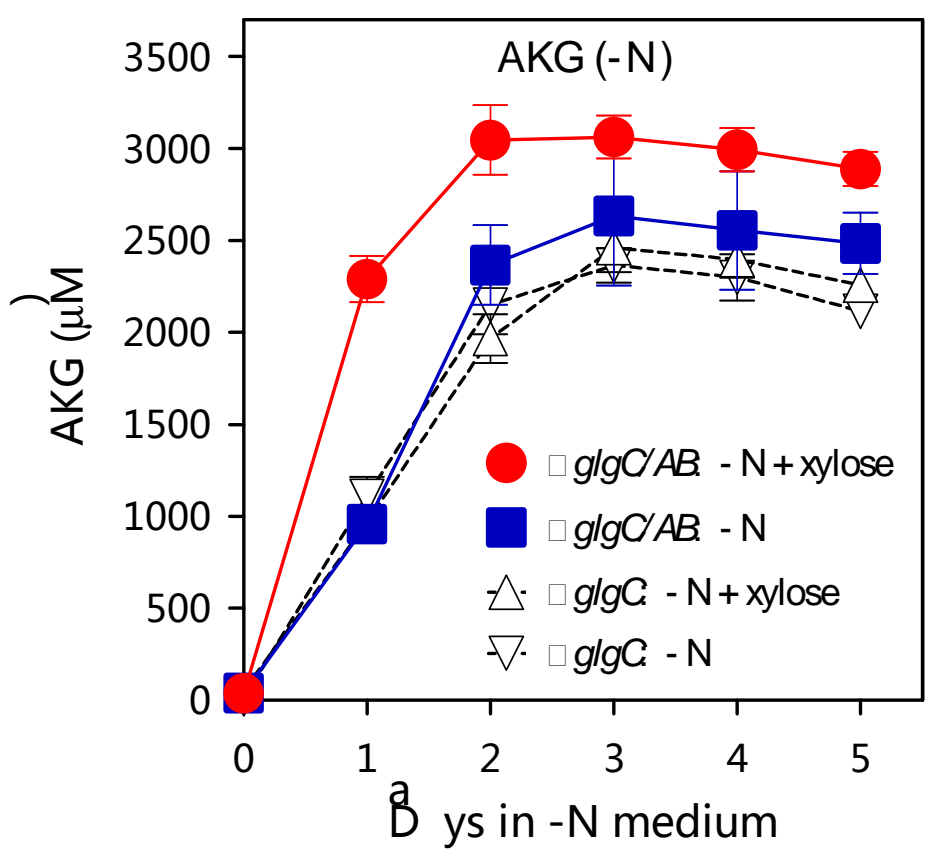

F

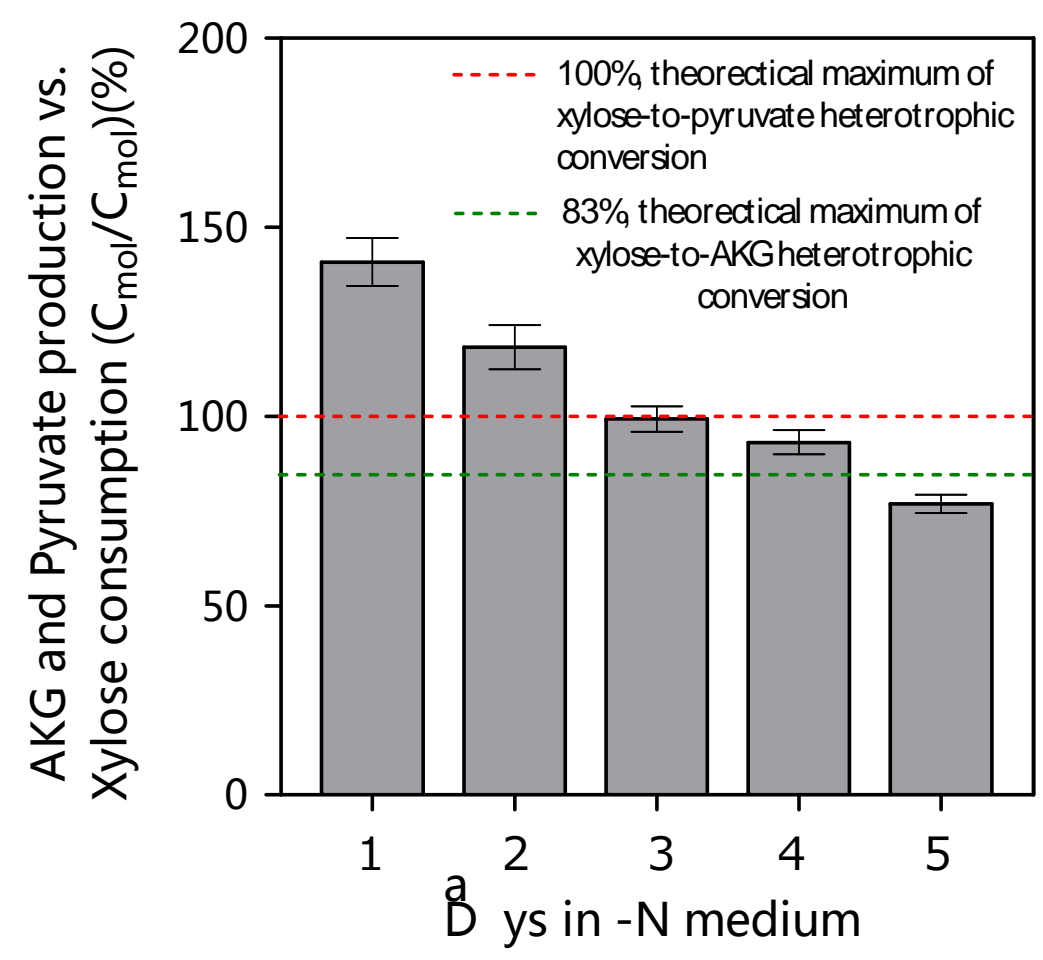


Fig. 6. Keto acid (AKG and pyruvate) production by mixotrophic cultures of Synechocystis, glycogen-storagedeficient mutant $(\Delta g l g C$ and $\Delta g l g C / x y l A B)$ strains. Cell growth $(+\mathrm{N}$ and $-\mathrm{N})(\mathbf{A})$, extracellular levels of pyruvate $(\mathbf{B}, \mathbf{D})$ and $A K G(C, E)$ produced by each strains under nitrogen sufficient $(+N)(A, B, C)$ or nitrogen depleted $(-N)$ $(A, D, E)$ conditions were analyzed. Photoautotrophic cultures of each strain in log phase $\left(\sim 0.8 \mathrm{OD}_{730}\right)$ were divided into controls (plotted as open triangle for $\Delta g \lg C$ and blue filled square for $\Delta g l g C / x y l A B$ respectively) and experimental groups supplemented with $20 \mathrm{mM}$ D-xylose (plotted as open triangle for $\Delta g / g C$ and red filled circle for $\Delta g l g C / x y \mid A B$, respectively). The conversion ratios of xylose to the keto acids by $\triangle g l g C / x y \mid A B$ strain under $-\mathrm{N}$ conditions were calculated (F) based on experimental data in $\mathbf{D}, \mathbf{E}$ (red closed cycles) and defined as the sum of the carbon molar quantities in the keto acids divided by carbon molar quantities of xylose that disappeared from the culture medium. The theoretical xylose-to-pyruvate and xylose-to-AKG yields in the heterotrophic mode are shown as red and green dotted lines, respectively. Symbols $\mathrm{N}$ and $\mathrm{X}$ represent nitrogen and D-xylose, respectively in (A). All concentration values of keto acids are given in $\mu \mathrm{M}$. Data with mean \pm standard deviations originate from three independent experiments.

heterotrophic mode) organic carbon utilization due to photosynthetic $\mathrm{CO}_{2}$ fixation. Taken together, these results support the notion that carbon excess from sugar (pentose or hexose) catabolism and/or reduced nutrient availability (nitrogen starvation) triggers metabolic overflow that results in the excretion of pyruvate and AKG in the absence of a major native carbon sink (glycogen). The nonbleaching phenotype reported for the glycogen synthesis mutant under photosynthetic, nitrogen starvation (-N) conditions (Carrieri et al., 2012) was also observed in $\triangle g l g C / x y / A B$ under mixotrophic (xylose supplementation), nitrogen starvation $(-\mathrm{N})$ conditions, indicating the blockage of 
phycobilisome degradation under both autotrophic and mixotrophic conditions by an unknown mechanism.

\subsection{Carbon flux is channeled from xylose into pyruvate and AKG}

To quantify the contribution of xylose utilization to keto acids production in a mixotrophic culture, we measured the percentages of carbon in pyruvate and AKG that are derived from xylose metabolism versus $\mathrm{CO}_{2}$ fixation with the isotope tracer experiment shown in Fig.7. The $\triangle g l g C / x y l A B$ strain was supplied with unlabeled D-xylose plus unlabeled bicarbonate (Fig. 7, A1), ${ }^{13} \mathrm{C}$-labeled bicarbonate only (Fig. 6, B1), or unlabeled xylose plus ${ }^{13} \mathrm{C}$-labeled bicarbonate (Fig. 7, C1). As such, the labeling pattern of the final product in each of the three cases can be deduced. Without a labeled carbon source, the pyruvate produced by $\triangle g l g C / x y \mid A B$ contained only ${ }^{12} \mathrm{C}$ (Fig. 7, A1). Exclusive assimilation of ${ }^{13} \mathrm{C}$-bicarbonate resulted in labelling of the pyruvate carbon atoms in all positions (Fig. 7, B1). When ${ }^{13} \mathrm{C}$-bicarbonate labeling was coupled to $\mathrm{U}-{ }^{12} \mathrm{C}$-D-xylose utilization, there was a mixture of unlabeled, partially labeled, and uniformly-labeled pyruvate (Fig. 7, C1). A molecule's fractional labeling enrichment (FL) can be calculated from mass spectroscopy data, and it represents the distribution of carbon originating from ${ }^{13} \mathrm{C}$-bicarbonate vs unlabeled carbon from D-xylose.

Fig. 7, A2-C3 shows isotope patterns of pyruvate and AKG as determined by LC-MS. As expected, single pyruvate and AKG peaks are shown as 87 and $145 \mathrm{~m} / \mathrm{z}$ in the control (Case A), respectively, indicating unlabeled pyruvate and AKG isotope upon ${ }^{12} \mathrm{C}-\mathrm{D}$-xylose and ${ }^{12} \mathrm{C}$-bicarbonate assimilation. In comparison, the highest values (Case B) were at $90(M+3)$ and $150 \mathrm{~m} / \mathrm{z}(\mathrm{M}+5)$ in the ${ }^{13} \mathrm{C}$ bicarbonate-supplied samples, suggesting that the majority of the pyruvate and AKG molecules were labeled uniformly in all carbon atoms. Minor isotopes of pyruvate (87, 88 and $89 \mathrm{~m} / \mathrm{z})$ and AKG (145, $146,147,148,149 \mathrm{~m} / \mathrm{z}$ ) represent unlabeled or partially labeled products, that could be derived from 
substrate impurity, biomass turnover, or unlabeled inorganic carbon that was initially present in the culture and medium. The $F L$ values show that most of the $C$ atoms are labeled in pyruvate $(86.8 \pm 3.9 \%)$ and $A K G(81.3 \pm 4.5 \%)$, confirming that they were derived from the inorganic carbon provided. These results are also consistent with previous NMR measurements (Carrieri et al., 2012) indicating that the pyruvate and AKG, secreted by the $\Delta g l g C$ mutant, originate mainly from de novo biosynthesis from photosynthetically-fixed $\mathrm{CO}_{2}$, rather than from remobilization of existing cellular materials. Supplementing the $\triangle g l g C / x y \mid A B$ strain with both ${ }^{13} \mathrm{C}$-bicarbonate and $\mathrm{U}-{ }^{12} \mathrm{C}$-D-xylose changed the labeling pattern significantly. Intermediate isotopes become dominant in both the pyruvate and AKG excreted, strongly implying the incorporation of unlabeled carbons from D-xylose. Due to the contribution of xylose utilization, much lower $F L$ are calculated (pyruvate, $45.3 \pm 4.7 \%$ and AKG, $37.3 \pm 0.6 \%$ ), indicating that as high as $~ 50 \%$ carbon ( $48 \%$ of the carbon in pyruvate and $54 \%$ in AKG) are derived from xylose utilization under the tested mixotrophic conditions.

\section{Discussion}

In this work, we expanded the substrate range in Synechocystis to include xylose via introduction of the E. coli xylose-utilization pathway, which not only allowed efficient LAHG and/or mixotrophic growth on xylose but also conferred tolerance to high xylose loading up to 200-300 mM during diurnal cycling. Engineering xylose assimilation also enhanced ethylene and keto acid production in photomixotrophic conversion, using both xylose and $\mathrm{CO}_{2}$ as the source of carbon simultaneously. Because the $\mathrm{CO}_{2}$ released from sugar metabolism can be recaptured by photosynthesis, theoretically all carbon in the sugar feedstock could eventually go into products by photomixotrophic conversion.

\subsection{Xylose transport}


Fig. 7

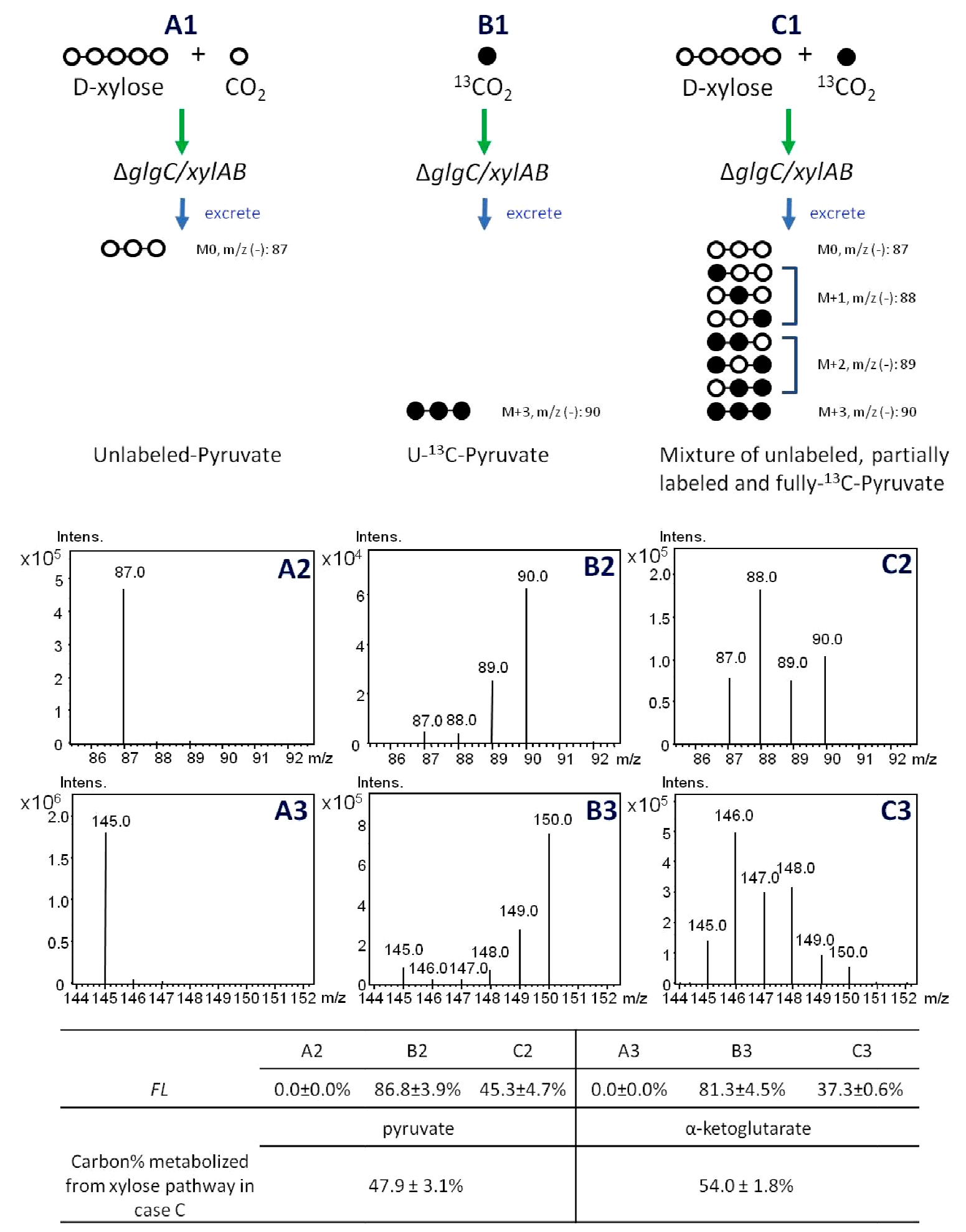


Fig. 7 Isotopic tracer and LC-MS analyses of pyruvate and AKG produced by the $\triangle g / g C / x y / A B$ strain, which concomitantly metabolizes (A) unlabeled D-xylose and unlabeled carbon dioxide, (B) ${ }^{13} \mathrm{C}$ labeled carbon dioxide only, and (C) unlabeled D-xylose plus ${ }^{13} \mathrm{C}$ labeled carbon dioxide. Predicted pyruvate isotope patterns resulting from different combinations of tracer substrate are shown in A1-C1. Closed cycles and open cycles, respectively, represent ${ }^{13} \mathrm{C}$-labled and unlabeled carbon atoms. $M 0, M+1, M+2$ and $M+3$ indicate that $0,1,2$ and 3 carbons are labeled in pyruvate. Also shown are the actual isotope patterns for pyruvate (panel A2-C2) and AKG (panel A3-C3) produced by the $\Delta g / g C / x y / A B$ culture labeled 24 hours with different tracer substrates. Fractional labeling enrichment $(F L)$ for isotopomers in each panel is shown in the bottom table ( $F L s$ were corrected for natural isotope abundance according to the approach described in Materials \& Methods). The carbon percentages metabolized from xylose pathway in case $\mathbf{C}$ was then determined with the following equation: $C_{\text {pyruvate }} \%$ from xylose $=1-\mathrm{FL}_{\mathrm{C} 2} / \mathrm{FL}_{\mathrm{B} 2}, \mathrm{C}_{\alpha \text {-ketoglutarate } \% \text { from xylose }}=1-\mathrm{FL}_{\mathrm{C} 3} / \mathrm{FL}_{\mathrm{B} 3}$. The data are from two independent experiments.

Since cyanobacteria were thought to be devoid of xylose transporters, a heterologous transporter was considered necessary for xylose uptake. Surprisingly, our results showed that the $x y I A B$ gene products $(\mathrm{XI}$ and $\mathrm{XK})$ in the engineered Synechocystis are sufficient to confer efficient xylose utilization without an additional heterologous xylose transporter, and that the downstream pathways are complete in this cyanobacterium (Fig. 1). Our data suggests that the presence of an endogenous xylose uptake system in Synechocystis probably involves the endogenous hexose-uptake system. The glucose facilitator, Glf, of Zymomonas mobilis, a member of the major facilitator superfamily (MFS), is known to transport fructose and xylose in addition to glucose (Saier and Crasnier, 1996). Interestingly, glcP, the only glucose transporter identified in Synechocystis and a member of the MFS, also possesses co-transport activity for fructose and glucose (Zhang et al., 1989). Whether or not 
this glcP is able to transport xylose remains to be studied.

\subsection{Xylose utilization augments photosynthetic ethylene production}

Our goal was to engineer a cyanobacterium to become a carbon-efficient, biological catalyst for the conversion of lignocellulosic sugars into value-added products. Building on photoautotrophic ethylene production in Synechocystis (Guerrero et al., 2012; Ungerer et al., 2012), we demonstrated that xylose utilization can increase ethylene productivity in both the light and dark, reaching a $64 \%$ increase over photoautotrophic condition at $40 \mu \mathrm{Em}^{-2} \mathrm{~s}^{-1}$ light (Fig. 5). Factors affecting ethylene production under photoautotrophic conditions have been identified previously, and include expression levels of EFE, nutrient supply, and light intensity (Ungerer et al., 2012). The current study shows that light availability is also a key environmental factor affecting ethylene production under photomixotrophic conditions. The current EFE level is a limiting factor for ethylene productivity, therefore improving EFE expression was expected to further increase mixotrophic ethylene production. In a closed photobioreactor, we have shown that biomass-derived sugars can provide substrate for ethylene production at night or supplement photosynthetic ethylene production during the day. Furthermore, $\mathrm{CO}_{2}$ produced from respiration and the ethylene-forming reaction might be reassimilated by photosynthesis so that more feedstock carbon is converted to ethylene.

\subsection{Xylose assimilation boosts the yield of organic acids}

In this study, xylose utilization significantly improved the excretion of keto acids (pyruvate and AKG) in photomixotrophic cultures of $\triangle g l g C / x y \mid A B$ over the photoautotrophic control (Fig. 6). Excretion of keto acids by glycogen storage mutants is thought to be the result of "metabolic overflow" in response to relative carbon excess resulting from glucose supplementation (under photomixotrophic conditions) and/or nitrogen starvation (Grundel et al., 2012). Under nitrogen replete conditions, keto acids can 
be excreted by $\triangle g l g C / x y \mid A B$ culture fed with xylose (Fig. 6 B, C), or in similar glycogen storage mutants fed with glucose (Grundel et al., 2012). Photomixotrophic growth and keto acid production patterns shown in this study are similar to those observed in certain heterotrophic microorganisms cultivated under carbon-excess conditions, during which keto acids were also the primary excreted products (Paczia et al., 2012). Overflow metabolism results in a variety of extracellular central metabolic intermediates and amino acids, and is considered a common feature of microorganisms during carbon-excess response (Paczia et al., 2012). Understanding the driving force and regulation of overflow metabolism in cyanobacteria could lead to new approaches for improving biofuels and bioproduct productivity. Under $\mathrm{N}$ starvation (-N), the keto acids productivity and the xylose-to-product conversion ratio decreases over time with or without xylose utilization (Fig. 6) (Carrieri et al., 2012). We have recently demonstrated that the decline in photosynthetic productivity can be reversed by the addition of limited nitrogen nutrient, thus renewing the bio-catalyst (Carrieri et al., 2015). It is expected that the same catalyst-regeneration scheme should also work under mixotrophic conditions.

Using isotope tracers, we estimated that the contribution of xylose utilization to de novo synthesis of pyruvate and AKG is roughly $50 \%$ under our experimental conditions. Given that each Rubisco reaction fixes one carbon, while the engineered xylose-utilization pathway incorporates five carbons for each reaction, the rate of xylose utilization is estimated to be $\sim 20 \%$ of the Rubisco influx. This rate is comparable with recent findings by (You et al. 2014) in which glucose was co-metabolized with $\mathrm{CO}_{2}$ by WT Synechocystis, and the glucose uptake rate was measured as $17.8 \pm 0.9 \%$ of Rubisco influx under mixotrophic conditions. These data suggest efficient import of xylose by native transporters, and efficient incorporation of xylose-derived carbon flux into central carbon metabolism under mixotrophic conditions. Taken together, these results demonstrate the potential of using biomass sugars to increase the productivity of cyanobacteria. 


\subsection{Concluding remarks}

Introduction of the $x y I A B$ genes from $E$. coli was necessary and sufficient to confer xylose-utilization capability into the model cyanobacterium, Synechocystis. The engineered strain demonstrated heterotrophic (LAHG)/photomixotrophic growth on xylose, and was able to tolerate high concentrations of xylose. Xylose utilization enabled the enhanced production of ethylene by a photomixotrophic process over that obtained through photosynthesis alone. Moreover, photosynthetic

productivity of two organic acids by a glycogen-synthesis mutant was significantly enhanced by xylose utilization. These increased productivities via improved carbon utilization $\left(\mathrm{CO}_{2}\right.$ or xylose) highlight the metabolic plasticity of Synechocystis. It also encourages further research on the efficient utilization of lignocellulosic feedstock by photosynthetic microbes capable of converting biomass sugars to fuels and chemicals without $\mathrm{CO}_{2}$ emission. Future research should aim at understanding the xylose transport mechanism in Synechocystis, and the interaction between photosynthesis and sugar utilization in diurnal cycles.

\section{Acknowledgements}

This work was supported by a Dragon-Gate grant (to TCL, IFC, HFC, SHJ) from National Council of Science (renamed Ministry of Science and Technology as of March 2014) in Taiwan. It was also supported in part by the U.S. Department of Energy, Office of Energy Efficiency and Renewable Energy, Fuel Cell Technologies Office (to PCM), BioEnergy Technologies Office (to JY), and NREL Director's Postdoc Fellowship (to WX). The authors acknowledge helpful discussion and technical assistance from Drs. Min Zhang, Yat-Chen Chou, Maria Ghirardi, Michael Seibert, Melissa Cano, and Carrie Eckert, all from NREL. 


\section{Author contributions}

TCL, SHJ, PCM and JY conceived the idea, analyzed data, and edited the manuscript. TCL, WX, TP,

DC, IFC, HFC and JU designed and performed the experiments, analyzed data, and wrote various parts of the manuscript. Funding sources had no role in these activities.

\section{References}

Adsul, M. G., Singhvi, M. S., Gaikaiwari, S. A., Gokhale, D. V., 2011. Development of biocatalysts for production of commodity chemicals from lignocellulosic biomass. Bioresour Technol. 102, 4304-12.

Anderson, S. L., McIntosh, L., 1991. Light-activated heterotrophic growth of the cyanobacterium Synechocystis sp. strain PCC 6803: a blue-light-requiring process. J Bacteriol. 173, 2761-7.

Angermayr, S. A., Hellingwerf, K. J., Lindblad, P., de Mattos, M. J., 2009. Energy biotechnology with cyanobacteria. Curr Opin Biotechnol. 20, 257-63.

Baebprasert, W., Jantaro, S., Khetkorn, W., Lindblad, P., Incharoensakdi, A., 2011. Increased H2 production in the cyanobacterium Synechocystis sp. strain PCC 6803 by redirecting the electron supply via genetic engineering of the nitrate assimilation pathway. Metab Eng. 13, 610-6.

Carrieri, D., Broadbent, C., Carruth, D., Paddock, T., Ungerer, J., Maness, P. C., Ghirardi, M., Yu, J., 2014. Enhancing photo-catalytic production of organic acids in the cyanobacterium Synechocystis sp. PCC 6803 $\Delta \mathrm{glgC}$, a strain incapable of glycogen storage. Microb Biotechnol. 8, 275-280.

Carrieri, D., Paddock, T., Maness, P. C., Seibert, M., Yu, J. P., 2012. Photo-catalytic conversion of carbon dioxide to organic acids by a recombinant cyanobacterium incapable of glycogen storage. Energy Environ. Sci. 5, 9457-9461.

Catalanotti, C., Yang, W., Posewitz, M. C., Grossman, A. R., 2013. Fermentation metabolism and its evolution in algae. Frontiers in plant science. 4, 150.

Davis, E. O., Henderson, P. J., 1987. The cloning and DNA sequence of the gene xylE for xylose-proton symport in Escherichia coli K12. J Biol Chem. 262, 13928-32.

Ducat, D. C., Way, J. C., Silver, P. A., 2011. Engineering cyanobacteria to generate high-value products. Trends Biotechnol. 29, 95-103.

Eckert, C., Xu, W., Xiong, W., Lynch, S., Ungerer, J., Tao, L., Gill, R., Maness, P. C., Yu, J., 2014. Ethyleneforming enzyme and bioethylene production. Biotechnol Biofuels. 7, 33.

Fan, L., Zhang, Y., Qu, W., Wang, J., Shao, W., 2011. Cloning and analysis of the xylAB operon and characterization of xylose isomerase from Thermoanaerobacter ethanolicus. Biotechnol Lett. 33, 593-8. 
Gao, Y., Xiong, W., He, M. J., Tang, L., Xiang, J. Y., Wu, Q. Y., 2009. Action spectra of chlorophyll a biosynthesis in cyanobacteria: dark-operative protochlorophyllide oxidoreductase-deficient mutants. Z Naturforsch C. 64, 117-24.

Grundel, M., Scheunemann, R., Lockau, W., Zilliges, Y., 2012. Impaired glycogen synthesis causes metabolic overflow reactions and affects stress responses in the cyanobacterium Synechocystis sp. PCC 6803. Microbiology. 158, 3032-43.

Guerrero, F., Carbonell, V., Cossu, M., Correddu, D., Jones, P. R., 2012. Ethylene synthesis and regulated expression of recombinant protein in Synechocystis sp. PCC 6803. PLoS One. 7, e50470.

Hahn-Hagerdal, B., Karhumaa, K., Fonseca, C., Spencer-Martins, I., Gorwa-Grauslund, M. F., 2007. Towards industrial pentose-fermenting yeast strains. Appl Microbiol Biotechnol. 74, 937-53.

He, Q., Vermaas, W., 1998. Chlorophyll a availability affects psbA translation and D1 precursor processing in vivo in Synechocystis sp. PCC 6803. Proc Natl Acad Sci U S A. 95, 5830-5.

Humbird, D., Davis, R., Tao, L., Kinchin, C., Hsu, D., Aden, A., Schoen, P., Lukas, J., Olthof, B., Worley, M., Sexton, D., Dudgeon, D., 2011. Process design and economics for biochemical conversion of lignocellulosic biomass to ethanol: dilute-acid pretreatment and enzymatic hydrolysis of corn stover (Figure 18: Mass, carbon, and energy balance diagram for the overall process). Technical report (NREL/TP-5100-47764) of NREL and Harris Group Inc.

Jeffries, T. W., 2006. Engineering yeasts for xylose metabolism. Curr Opin Biotechnol. 17, 320-6.

Jojima, T., Omumasaba, C. A., Inui, M., Yukawa, H., 2010. Sugar transporters in efficient utilization of mixed sugar substrates: current knowledge and outlook. Appl Microbiol Biotechnol. 85, 471-80.

Kim, S. R., Ha, S. J., Wei, N., Oh, E. J., Jin, Y. S., 2012. Simultaneous co-fermentation of mixed sugars: a promising strategy for producing cellulosic ethanol. Trends Biotechnol. 30, 274-82.

Kong, R., Xu, X., Hu, Z., 2003. A TPR-family membrane protein gene is required for light-activated heterotrophic growth of the cyanobacterium Synechocystis sp. PCC 6803. FEMS Microbiol Lett. 219, 75-9.

Lam, M. K., Lee, K. T., 2012. Microalgae biofuels: A critical review of issues, problems and the way forward. Biotechnol Adv. 30, 673-90.

Lange, J. P., van der Heide, E., van Buijtenen, J., Price, R., 2012. Furfural--a promising platform for lignocellulosic biofuels. ChemSusChem. 5, 150-66.

Lawford, H. G., Rousseau, J. D., 1994. Relative rates of sugar utilization by an ethanologenic recombinant Escherichia coli using mixtures of glucose, mannose, and xylose. Appl Biochem Biotechnol. 45-46, 367-81.

Lindberg, P., Park, S., Melis, A., 2010. Engineering a platform for photosynthetic isoprene production in cyanobacteria, using Synechocystis as the model organism. Metab Eng. 12, 70-9. 
Lu, X., 2010. A perspective: photosynthetic production of fatty acid-based biofuels in genetically engineered cyanobacteria. Biotechnol Adv. 28, 742-6.

Luo, B., Groenke, K., Takors, R., Wandrey, C., Oldiges, M., 2007. Simultaneous determination of multiple intracellular metabolites in glycolysis, pentose phosphate pathway and tricarboxylic acid cycle by liquid chromatography-mass spectrometry. J Chromatogr A. 1147, 153-64.

Machado, I. M., Atsumi, S., 2012. Cyanobacterial biofuel production. J Biotechnol. 162, 50-6.

Makarova, O., Kamberov, E., Margolis, B., 2000. Generation of deletion and point mutations with one primer in a single cloning step. Biotechniques. 29, 970-2.

McEwen, J. T., Machado, I. M., Connor, M. R., Atsumi, S., 2013. Engineering Synechococcus elongatus PCC 7942 for Continuous Growth under Diurnal Conditions. Appl Environ Microbiol. 79, 1668-75.

Meijnen, J. P., de Winde, J. H., Ruijssenaars, H. J., 2008. Engineering Pseudomonas putida S12 for efficient utilization of D-xylose and L-arabinose. Appl Environ Microbiol. 74, 5031-7.

Nanchen, A., Fuhrer, T., Sauer, U., 2007. Determination of metabolic flux ratios from 13C-experiments and gas chromatography-mass spectrometry data: protocol and principles. Methods Mol Biol. 358, 177-97.

Paczia, N., Nilgen, A., Lehmann, T., Gatgens, J., Wiechert, W., Noack, S., 2012. Extensive exometabolome analysis reveals extended overflow metabolism in various microorganisms. Microb Cell Fact. 11, 122.

Rogers, P. L., Jeon, Y. J., Lee, K. J., Lawford, H. G., 2007. Zymomonas mobilis for fuel ethanol and higher value products. Adv Biochem Eng Biotechnol. 108, 263-88.

Saha, B. C., 2003. Hemicellulose bioconversion. J Ind Microbiol Biotechnol. 30, 279-91.

Saier, M. H., Jr., Crasnier, M., 1996. Inducer exclusion and the regulation of sugar transport. Res Microbiol. 147, 482-9.

Savakis, P. E., Angermayr, S. A., Hellingwerf, K. J., 2013. Synthesis of 2,3-butanediol by Synechocystis sp. PCC6803 via heterologous expression of a catabolic pathway from lactic acid- and enterobacteria. Metab Eng. 20, 121-30.

Shahzadi, T., Mehmood, S., Irshad, M., Zeeshan, N., Rashid, U., Sughra, K., 2014. Advances in lignocellulosic biotechnology: A brief review on lignocellulosic biomass and cellulases. Ad in Biosci Biotech. 5, $246-251$.

Tabei, Y., Okada, K., Makita, N., Tsuzuki, M., 2009. Light-induced gene expression of fructose 1,6bisphosphate aldolase during heterotrophic growth in a cyanobacterium, Synechocystis sp. PCC 6803. FEBS J. 276, 187-98.

Toivari, M. H., Aristidou, A., Ruohonen, L., Penttila, M., 2001. Conversion of xylose to ethanol by recombinant Saccharomyces cerevisiae: importance of xylulokinase (XKS1) and oxygen availability. Metab Eng. 3, $236-49$.

Ungerer, J., Tao, L., Davis, M., Ghirardi, M., Maness, P., Yu, J., 2012. Sustained photosynthetic conversion of CO2 to ethylene in recombinant cyanobacterium Synechocystis 6803. Energy Environ. Sci. 5, 8998-9006. 
Ungerer, J. L., Pratte, B. S., Thiel, T., 2008. Regulation of fructose transport and its effect on fructose toxicity in Anabaena spp. J Bacteriol. 190, 8115-25.

van Maris, A. J., Winkler, A. A., Kuyper, M., de Laat, W. T., van Dijken, J. P., Pronk, J. T., 2007. Development of efficient xylose fermentation in Saccharomyces cerevisiae: xylose isomerase as a key component. Adv Biochem Eng Biotechnol. 108, 179-204.

Vishnu Menon, M. R., 2012. Trends in bioconversion of lignocellulose: Biofuels, platform chemicals \& biorefinery concept. Progress in Energy and Combustion Science 38, 522-550.

Yu, Y., You, L., Liu, D., Hollinshead, W., Tang, Y. J., Zhang, F., 2013. Development of Synechocystis sp. PCC 6803 as a phototrophic cell factory. Mar Drugs. 11, 2894-916.

Zhang, C. C., Durand, M. C., Jeanjean, R., Joset, F., 1989. Molecular and genetical analysis of the fructoseglucose transport system in the cyanobacterium Synechocystis PCC6803. Mol Microbiol. 3, 1221-9.

Zhang, M., Eddy, C., Deanda, K., Finkelstein, M., Picataggio, S., 1995. Metabolic Engineering of a Pentose Metabolism Pathway in Ethanologenic Zymomonas mobilis. Science. 267, 240-3.

Zhou, J., Zhang, H., Zhang, Y., Li, Y., Ma, Y., 2012. Designing and creating a modularized synthetic pathway in cyanobacterium Synechocystis enables production of acetone from carbon dioxide. Metabolic Engineering $14,394-400$. 